\title{
An Enhanced Optical Flow Technique for Radar Nowcasting of Precipitation and Winds
}

\author{
RENZO BECHINI, ${ }^{\mathrm{a}}$ AND V. CHANDRASEKAR \\ Colorado State University, Fort Collins, Colorado
}

(Manuscript received 22 June 2017, in final form 15 September 2017)

\begin{abstract}
The atmospheric state evolution is an inherently highly complex three-dimensional problem that numerical weather prediction (NWP) models attempt to solve. Although NWP models are being successfully employed for medium- and long-range forecast, their short-duration forecast (or nowcast) capabilities are still limited because of model initialization challenges. On the lower end of the complexity scale, nowcasting by extrapolation of two-dimensional weather radar images has long been the most effective tool for nowcasting precipitation. Attempts are being made to take advantage of both approaches by blending extrapolation and numerical model forecasts. In this work a different approach is presented, relying on the additional Doppler radar wind information and a simplified modeling of basic physical processes. Instead of mixing the outputs of different forecasts as in blended approaches, the idea behind this study is to combine extrapolation and precipitation modeling in a new technique with a higher level of complexity with respect to conventional nowcasting methods, although still much simpler than NWP models. As a preliminary step, the Variational Doppler Radar Analysis System (VDRAS) is used to provide an initial analysis exploiting all the available dual-polarization and Doppler radar observations. The rainwater and wind fields are then advected using an optical flow technique that is subject to simplified physical interactions. As a result precipitation and wind nowcasting are obtained and are successively validated up to a 1-h lead time, showing potential improvement upon standard extrapolation.
\end{abstract}

\section{Introduction}

The description of the atmospheric state evolution is inherently a three-dimensional problem involving several variables (e.g., for warm rain process: temperature, humidity, pressure, winds, water vapor, cloud water, rainwater). However, nowcasting by radar is in general intended as the two-dimensional forecast of rainwater $q_{r}$ $\left(\mathrm{g} \mathrm{kg}^{-1}\right)$ in the near-surface layer. One possible option for precipitation and wind nowcasting is through the use of numerical weather prediction (NWP) models on a limited domain, and considering a specific section of the forecasted atmospheric state, that is, three variables (a precipitation-related quantity and the two wind components) on a single horizontal layer. While data assimilation is contributing to improving the skill of NWP models at short lead times (Ballard et al. 2016), over a forecast range on the order of $\sim 60 \mathrm{~min}$ the model

${ }^{\mathrm{a}}$ Current affiliation: Arpa Piemonte, Turin, Italy.

Corresponding author: Renzo Bechini, r.bechini@arpa. piemonte.it forecast typically shows poorer performance with respect to methods based on the simple extrapolation of the observed reflectivity or related quantity (Germann and Zawadzki 2002; Hwang et al. 2015).

Extrapolation-based precipitation nowcasting exclusively relies on one variable (typically the radar reflectivity) observed on a given surface, for example, the conical scan surface. Nowcasting by extrapolation often makes use of optical flow techniques to estimate the motion vectors based on two or more previous observations. The motion vectors are then used to advect in time the most recent observations. Given the huge difference in terms of complexity between the model and the extrapolation approaches, the performance attainable by adopting the latter technique for precipitation nowcasting is somewhat surprising. The reason is that in general the advective component of the storm evolution prevails over the more complex dynamical and microphysical interactions within the storm and with the surrounding environment. Extrapolation-based methods provide good results for very short-term forecasting (up to about $20 \mathrm{~min}$ ), but their performance tends to quickly 
decrease as result of the absence of a representation of the atmospheric physics, more specifically:

- There is a lack of source/sink terms: the most recent observations are simply advected, assuming Lagrangian persistence, irrespective of possible growth or decay affecting the storm evolution.

- The motion vectors are estimated from observations typically confined over a portion of the radar domain. When the storms are advected in regions originally not showing radar echoes, the motion vector estimates may lose their representativeness.

The different skill at increasing lead times of extrapolation and NWP models led to the development of blending techniques, with the aim to take advantage of both methods (Seed et al. 2013; Hwang et al. 2015). In this work a different approach is presented to attempt to improve the skill of standard extrapolation methods, including the additional Doppler radar wind observations and a simplified representation of the convective precipitation process.

The use of a four-dimensional data assimilation system has the potential to provide an accurate low-level wind analysis. Unlike simpler multiple-Doppler techniques, the adoption of a cloud-scale numerical model in the assimilation process ensures the physical consistency between the wind and the rainwater fields over the assimilation time window. The availability of a reliable estimation of the low-level wind field may help to compensate the previously mentioned limitations of standard extrapolation methods. In particular, the divergence of the lowlevel winds provides useful indications about the location of convergence (negative divergence) regions near the surface, where intensification of an existing storm or a new development is more likely. On the other hand, the downdraft inferred from the positive divergence regions, in conjunction with the rainwater content evolution, may be used to attempt a simple representation of the outflow that is relevant to the evolution of the wind field in the lower level (Cotton et al. 2010).

"Mean winds" methods have also been used with some success to predict the storm motion. These methods rely on the empirical evidence that the motion of the storms correlates well with the average wind in the low to midtroposphere. For example, Davies and Johns (1993) used the average wind in the lowest $0-6 \mathrm{~km}$ AGL. The rainwater motion vectors estimated using the past storm location can therefore be regarded as representative of some weighted average of the lower tropospheric winds. It seems then consistent to attempt to exploit the low-level wind prediction to update the initial estimate of the rainwater motion vectors during the forecasting phase, assuming that the upper level winds will evolve more slowly.
This work represents an attempt to model the interconnections between the rainwater and the low-level wind evolution, relying on the independent estimation of the motion vectors for the rainwater and for the components of the wind field (divergence and vorticity). The proposed technique is applied over the Dallas-Fort Worth (DFW), Texas, region, where measurements from multiple radars (NEXRAD and X-band systems) are available within the DFW Urban Demonstration Network. The scanning strategy of the small X-band radars is inherited from the Center for Collaborative Adaptive Sensing of the Atmosphere (CASA) project's distributed collaborative adaptive sensing concept (Junyent et al. 2010) and is intended to sample with high time resolution the lower atmosphere (1-3 km AGL).

The paper is organized as follows. Section 2 illustrates the analysis method based on a four-dimensional variational assimilation of radar observations, while section 3 describes the optical flow technique employed for estimating the motion vectors from a pair of consecutive analyses. The parametric model for precipitation and wind nowcasting is discussed in section 4 , and the achieved results for a representative case study are presented in section 5. Finally, the main findings are summarized and discussed in section 6 .

\section{Analysis}

Four-dimensional data assimilation techniques have been introduced during the last couple of decades in global and regional models (Rabier et al. 2000; Rawlins et al. 2007; Tanguay et al. 2012) to provide a temporal extension with respect to previous three-dimensional variational systems. In large-scale models, the fourdimensional assimilation process is mainly intended as an optimal filter, but for the convective scale it becomes fundamental to retrieve the unobserved variables. In fact, while for the mesosynoptic scale the conventional meteorological observations (surface measurements and upper-air soundings) provide adequate resolution to represent the model variables, for the convective scale only weather radars are able to collect observations with enough spatial and temporal resolution, although for a reduced set of variables. In addition, the variables observed by weather radar are not among the model variables but are generally related to the water content and winds through appropriate observation operators.

The Variational Doppler Radar Analysis System (VDRAS) is an advanced data assimilation system specifically designed for ingesting Doppler weather radar observations at the convective scale (Sun and Crook 1997, 1998). The system has been installed at many sites around the world and is typically running using long-range 



FIG. 1. PPI from the Midlothian radar at 2039 UTC: (a) reflectivity, (b) hydrometeor classification, and (c) rainwater mixing ratio estimated from dual-polarization observations.

operational radar networks. The core four-dimensional data assimilation scheme is based on a cloud-scale model and typically considers a 15-20-min time window for the radar assimilation, with $1-3-\mathrm{km}$ spatial resolution. The analysis in this study is performed using VDRAS over the DFW region (Bechini et al. 2015).

\section{a. Radar data preprocessing}

The rainwater mixing ratio is conventionally derived in VDRAS from reflectivity observations using a powerlaw relation obtained assuming a Marshall-Palmer raindrop size distribution:

$$
Z_{h}=43.1+17.5 \log _{10}\left(\rho q_{r}\right),
$$

where $Z_{h}$ is the horizontal reflectivity $(\mathrm{dB} Z)$ and $\rho$ is the air density. However, for X-band systems path attenuation greatly affects the reliability of reflectivity-based estimates in heavy precipitation. Dual-polarization measurements allow for correcting path attenuation and for estimating the rain rate and the rainwater content with higher accuracy (Bringi and Chandrasekar 2001).

In this work a blended algorithm is adopted that combines the available dual-polarization observations in addition to $Z_{h}$ (namely, the differential reflectivity $Z_{\mathrm{dr}}$ and the specific differential phase shift $K_{\mathrm{dp}}$ ) using different relations, providing a rainwater estimate less sensitive to drop size distribution (DSD) variations and mostly unaffected by attenuation. The basis for applying different relations is provided by a preliminary hydrometeor classification (Bechini and Chandrasekar 2015) and according to predefined thresholds on the dual-polarization variables (Cifelli et al. 2011). The relations used for the liquid water content (LWC)that is, the rainwater content mixing ratio scaled by air density $\left(\mathrm{LWC}=\rho q_{r}\right)$-include functions of $Z_{h}$ and $Z_{\mathrm{dr}}$,
$K_{\mathrm{dp}}$ and $Z_{\mathrm{dr}}$, and $K_{\mathrm{dp}}$ only, with coefficients as in Bringi and Chandrasekar (2001).

For assimilation in VDRAS, the radar observations of radial Doppler velocity $v_{r}^{\text {obs }}$ and the estimates of $q_{r}^{\text {obs }}$ in the polar domain are first interpolated to Cartesian PPIs with 500-m resolution. Figure 1 shows an example of a reflectivity PPI at $2.0^{\circ}$ elevation from the Midlothian, Texas, X-band radar, with hydrometeor classification and the resulting $q_{r}^{\text {obs }}$ estimation.

\section{b. Model setup}

For the hailstorm event that occurred on 12 May 2014, the data from the S-band NEXRAD KFWS (DFW) radar have been considered, in addition to the X-band radars located in Arlington, Texas, and Midlothian. To provide an initial condition (background) for the cost function minimization in VDRAS, a mesoscale analysis is preliminarily performed to start the model simulation (cold start), based on surface observations (METARs) and vertical sounding from a preliminary WRF Model simulation on a larger domain. The experiment started at 1932UTC and then cycled every $\sim 5 \mathrm{~min}$, matching with the NEXRAD volume update frequency (292s) and using the previous forecast as background. This ensures the availability of large-scale three-dimensional coverage for the analysis. The assimilation window is set to $3 \mathrm{~min}$, allowing for the inclusion of one S-band volume scan and three to five low-level PPI scans from each X-band system. In previous VDRAS applications, assimilation windows of about 15-20 min have been used, including several radar volume scans. The reference time for all the observations during the whole volume scan was the beginning of the first elevation scan, disregarding the time differences between successive elevations. In the current setup, however, a short assimilation window is required to consider the actual observation time of the individual 


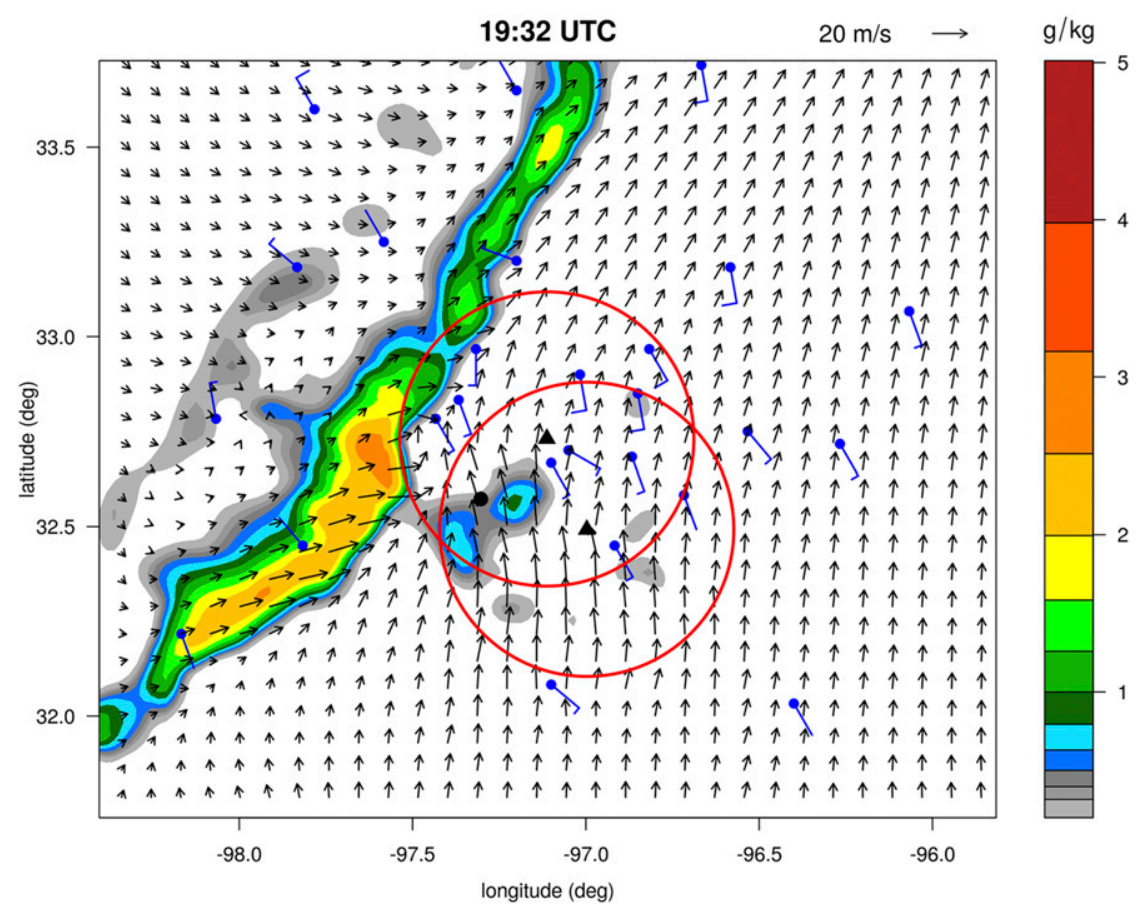

FIG. 2. VDRAS analysis at the 600-m vertical level of $q_{r}$ (colors) and winds at 1932 UTC from the first cycle on the full model domain. Shown are the METAR surface observations (blue wind barbs), the $40 \mathrm{~km}$-range domain (red circles) of the two X-band radar (black triangles), and the position of the NEXRAD KFWS radar (filled black circle).

PPIs within the NEXRAD volume scan, in order to deal more consistently with the frequent low-level scans of the $\mathrm{X}$-band systems.

The model domain is $122 \times 112 \times 30(n x, n y, n z$, respectively) grid points, with a horizontal resolution of $2 \mathrm{~km}$, a vertical resolution of $400 \mathrm{~m}$, and an integration time step of $4 \mathrm{~s}$. These parameters, in combination with the short assimilation window, allow for reducing the wall-clock time for the generation of a single analysis to about $5 \mathrm{~min}$, using 16 processors.

In Fig. 2 the VDRAS analysis from the first (cold start) four-dimensional variational data assimilation (4D-Var) cycle is shown on the full model domain. At this time, a squall line can be seen approaching from west-northwest within the DFW region covered by the X-band radars (red circles). The wind analysis during the following period can then rely on multiple Doppler observations and on several surface METAR measurements.

\section{Motion vectors estimation}

Many techniques exist to estimate the motion of objects or surfaces from a sequence of ordered images. These techniques are generally referred to as optical flow and can rely on different methods for the determination of the motion. In the atmospheric sciences, besides weather radar, important developments in optical flow techniques originate from lidar (Hamada et al. 2016; Dérian et al. 2015) and satellite (Velden et al. 2005; Heas and Memin 2008) applications. For weather radar, the most popular approaches are the block-based methods, minimizing the sum of squared differences or maximizing the normalized cross correlation (Rinehart and Garvey 1978; Chornoboy et al. 1994) or the variational methods (Germann and Zawadzki 2002). Horn and Schunck (1981) were the first to propose a variational method for optical flow estimation. In their seminal work, the basic optical flow constrain equation, frequently referred as the brightness constancy assumption, states that the apparent brightness $I$ of moving objects remains constant over time. This is expressed as

$$
\frac{d I}{d t}=\nabla I \cdot \mathbf{u}(\mathbf{x})+\frac{\partial I}{\partial t}=0
$$

where $\mathbf{u}=(u, v)$ is the unknown motion vector field on the $\mathbf{x}=(x, y)$ plane. This equation cannot be solved pointwise. In fact, because of the two unknowns, only the magnitude of the motion in the gradient direction can be estimated. To solve this aperture problem, some additional constraints need to be introduced. Horn and Schunck proposed a variational method with global 
smoothing to ensure filling in the motion estimate from nearby gradient constraints. The variational problem is thus solved by minimizing an energy functional,

$$
J=\iint\left[\left(\frac{\partial I}{\partial x} u+\frac{\partial I}{\partial y} v+\frac{\partial I}{\partial t}\right)^{2}+\alpha^{2}\left(|\nabla u|^{2}+|\nabla v|^{2}\right)\right] d x d y,
$$

where the parameter $\alpha$ is a regularization constant to control the smoothness of the motion estimates. Larger values of $\alpha$ lead to a smoother flow. The minimization of $J$ can be achieved by solving the associated EulerLagrange equations. The method relies on the proper estimation of the partial derivatives $\partial I / \partial x, \partial I / \partial y$, and $\partial I / \partial t$. If these cannot be correctly estimated because of highly nonlinear gradients or excessively large displacements of the precipitation patterns between successive images, then the motion vectors cannot be correctly calculated. To overcome the possible issue related to nonlinear gradients, the radar reflectivity (or logarithmic rainwater) at a given vertical level is considered for the estimation of the motion vectors. In fact, the rainfall rate (or liquid water) tends to show high peaks with exponential decay away from the precipitation core in convective precipitation. On the other hand, logarithmic quantities like reflectivity present a more linear decay. The second issue may arise from either excessive physical displacements or a too high grid resolution. To overcome this possible issue, the multiscale strategy approach of MeinhardtLlopis et al. (2013) is adopted. In their method a pyramidal structure provides a coarse-to-fine successive refinement of the flow field. The input reflectivity images are filtered and downsampled by a factor $\eta$ using bicubic interpolation. Starting at the coarsest scale, the optical flow equations are solved and every intermediate solution is used as the initialization in the next scale. The $\eta$ and the number of scales $N$ are chosen based on the expected maximum storm motion, the grid resolution of the images, and the time lag between two successive images, in order to keep the motion to be detected small at the coarsest scale. For the case discussed in section 5 , the values $\eta=0.5$ and $N=4$ are used.

Figure 3 shows an example of motion vector estimation for the case study discussed in section 5 , for the rainwater (Figs. 3a,c) and the divergence field obtained from the VDRAS wind analysis (Figs. 3b,d).The motion vectors for the rainwater show a dominant westerly component on the squall line near the KFWS radar (moving eastward), while a southwesterly flow is estimated ahead of the storm. This is in fair agreement with the VDARS wind retrieval in the low levels (Fig. 2). In this specific case, the optical flow method is able to derive a reliable estimate ahead of the storm, exploiting the motion of the two small cells close to the two X-band radars (triangle symbol). The divergence (Figs. 3b,d) shows a westerly flow over most of the domain, also ahead of the storm, with a small modulation across the squall line. The motion fields of rainwater and the wind components calculated from the VDRAS wind analysis (divergence and vorticity) may show relevant differences that are expected to bring useful complementary information to the forecast.

\section{a. Semi-Lagrangian advection}

After the estimation of the motion vectors, the advection of the rainwater and wind components can be accomplished using either forward or backward schemes. Forward (in time) schemes foresee the distribution of the advected quantity among the neighboring grid points around the destination point (which in general does not coincide with a grid point). If the flow is divergent, however, this method may lead to "holes" in the advected map. Another approach is to use backward advection; that is, for a given grid point, the origin at the previous time step is found by following the flow backward. This again will not coincide with a grid point, so in this case interpolation is necessary (Germann and Zawadzki 2002). Bilinear interpolation is often used for this purpose. However, in order to reduce the diffusion arising from the bilinear scheme, a bicubic interpolation is adopted here.

The combination of the Lagrangian perspective and the use of a regular grid Eulerian framework is known as the semi-Lagrangian scheme. This class of methods has the notable advantage of being particularly efficient, allowing for the use of large time steps.

The backward scheme is in general not mass conservative, although it may be considered nearly mass conservative when the divergence of the flow field is negligible. So, in order to ensure mass conservation, the divergence component of the rainwater motion vectors field needs to be removed, or at least severely damped. This is accomplished by relying on a technique widely used in fluid dynamics simulations (Stam 1999). According to Helmholtz's theorem, the wind vector field can be decomposed into an irrotational component and a nondivergent component. For a horizontal wind vector $\mathbf{V}$, the vertical vorticity $\xi$ and divergence $\delta$ are defined as

$$
\xi=\mathbf{k} \cdot \nabla \times \mathbf{V}=\frac{\partial v}{\partial x}-\frac{\partial u}{\partial y}, \quad \delta=\nabla \cdot \mathbf{V}=\frac{\partial u}{\partial x}+\frac{\partial v}{\partial y} .
$$

The wind field can then be expressed in terms of the streamfunction $\psi$ and velocity potential $\chi$ : 

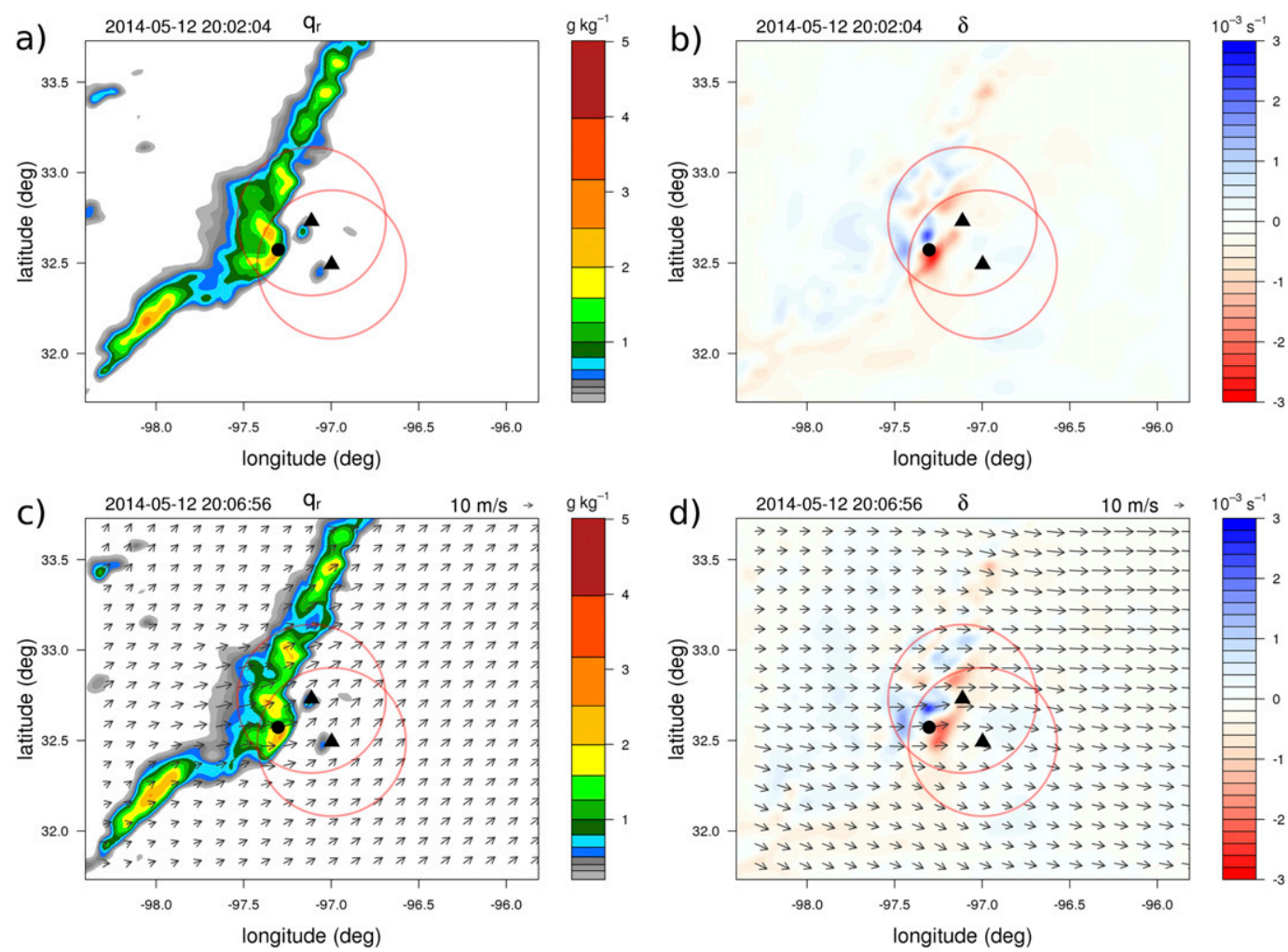

FIG. 3. Example of motion vector estimations. (a),(c) Rainwater analysis at 600-m height for two successive time steps (2002 and 2006 UTC). Based on the optical flow solution on this pair of images, the motion vectors in (c) are estimated. (b),(d) Corresponding motion vector estimations for the divergence fields at the same vertical level as in (a),(c). Only one vector every six grid points is plotted for clarity.

$$
\mathbf{V}=\mathbf{V}_{\psi}+\mathbf{V}_{\chi}=\nabla \chi+\mathbf{k} \times \nabla \psi
$$

From Eq. (4) and taking the vertical component of the curl and the divergence of Eq. (5), the Poisson equations for $\xi$ and $\delta$ are obtained:

$$
\nabla^{2} \psi=\xi, \quad \nabla^{2} \chi=\delta
$$

The divergent component of the motion vectors can then be subtracted in three steps:

- calculate divergence from the motion vector field

- solve for the velocity potential $\chi$, given that $\nabla^{2} \chi=\delta$, using a iterative successive overrelaxation (SOR) technique

- subtract $\nabla \chi$ from the original motion vector field

This procedure is especially important when the motion vector field is mixed with the low-level wind field during the forecast, as described later in section 4c. In fact the low-level wind field generally has a quite relevant divergence component, which would severely impact the stability of the rainwater advection in terms of mass conservation, causing unphysical visual deformations (excessive stretching/ shrinking).

\section{b. Image registration}

As described in the next section, the parametric model relies on the analyses at two vertical levels for the estimation of the rainwater content gradient. The vertical gradient is used to estimate the contribution to the rainwater in the lowest level by vertical advection. However, possible tilting of the storm may affect this estimate by introducing artifact gradients. In fact, the two-dimensional model does not resolve the vertical wind shear, so the high-level rainwater needs to be aligned with the low-level field to compensate for the eventual tilting. In addition, depending on the scanning strategy and the analysis method, an apparent additional tilting may be introduced by the delayed radar scanning of the higher elevations. Correction of the apparent misalignment can be performed through image registration. Optical flow may also be used for this purpose, so the Horn-Schunck technique described in section 3 is also applied to determine the appropriate deformation (motion vectors) to align the upper-level rainwater field 
to the lower-level rainwater field. In this preliminary evaluation of the method, the same optical flow parameters used for the motion vector estimation of the rainwater and wind components are adopted, specifically the regularization parameter $\alpha=80$. This large value of $\alpha$ (smooth displacement) may lead to an overestimation of the vertical gradients in some cases, although the overall impact on the quality of the forecast appears limited. Further investigation will be devoted to assessing the impact of tilting in different conditions and sensitivity to the optical flow settings.

\section{Parametric model for nowcasting}

If the two basic assumptions for the optical flow-that is, the stationarity of the motion vectors and the lack of a source term-are removed, then Eq. (2) becomes

$$
\frac{d q_{r}}{d t}=\nabla q_{r} \cdot \mathbf{u}(t, \mathbf{x})+\frac{\partial q_{r}}{\partial t}=S(t, \mathbf{x}) .
$$

If the level of brightness is not constant, then the motion estimate can be biased. To cope with this limitation, methods like the integrated continuity equation (ICE) have been developed and applied to satellite imagery (Fitzpatrick 1988; Corpetti et al. 2002; Heas et al. 2007). Other attempts have also been made to develop methods including brightness variation caused by time-dependent physical models (Haussecker and Fleet 2001), although these were mainly limited to relatively simple applications, such as changing illumination or thermal diffusion in infrared images.

The approach adopted here is based on a separate treatment of the optical flow derived from Eq. (2) and the local rate of change of the rainwater content and winds. The proposed model relies on two consecutive analyses from two vertical levels of the VDRAS assimilation system:

- $600 \mathrm{~m}$ MSL (rainwater and winds)

- 3400 m MSL (rainwater).

The choice of the specific levels is dictated by the need to have a sufficient vertical spacing inside the liquid phase layer to calculate a reliable rainwater gradient (section 4a). While the VDRAS analysis provides the full set of atmospheric variables, only the radar observable fields (rainwater and winds) are considered in this approach. The idea is to constrain the observations (analyses) using a simplified physical model with adaptive parameters. It is argued that while the deficient physical description will inherently limit the validity of the forecast for large lead times, the adaptiveness of the model may help to improve the very short-term forecast (0-60 min). The traditional use of extrapolation applied to reflectivity (rainwater) is here extended to the wind components, and simple relations governing the rain growth and decay are defined and heuristically tuned through a set of adaptive parameters.

In an observational environment such as the DFW test bed, the architecture of the weather radar network provides the best coverage in the atmospheric layer closer to the surface. From this perspective, the aim of the proposed approach is to extract the most valuable information content from the available observations. In addition to the rainwater content, the analysis and nowcast of the wind field near the surface have a special relevance of their own for the potential impact on human activities and infrastructures. A suitable representation of the dynamics taking place in the lowest layer is also important for the tight relation with the storm evolution.

The basic steps of the parametric model are described in detail in the following subsections. The divergence and vorticity are initially calculated from the low-level wind analysis. Considering a pair of observational time frames, typically lag-0 (time $\left.t_{0}\right)$ and lag- 1 (time $t_{0}-1$ ), the optical flow technique is applied independently to $q_{r}$, $\delta$, and $\xi$, obtaining the motion vectors $\mathbf{U}_{q r}, \mathbf{U}_{\delta}$, and $\mathbf{U}_{\xi}$, respectively. The divergence and vorticity are advected to the next time step using the respective motion vectors, while before applying advection to $q_{r}$, its local rate of change at the two vertical levels is estimated (section 4a).

\section{a. Growth and decay parameterization}

The parameterization of the growth and decay local rate of change is realized according to the following equations:

$$
\begin{aligned}
\frac{\partial q_{r}}{\partial t} & =\frac{\partial q_{r}}{\partial z}\left(w+V_{t}\right)+p_{0} w, \quad \text { and } \\
V_{t} & =5.40 q_{r}^{0.125}
\end{aligned}
$$

where $V_{t}$ is the terminal fall velocity of raindrops (Sun and Crook 1997), $p_{0}$ is the condensation parameter (Table 1), and $w$ is the vertical velocity of air obtained from the divergence in the hypothesis of mass conservation. Since the divergence at the near-surface level $(600 \mathrm{~m})$ is being considered, assuming $w=0$ below this level and zero divergence at the upper level $(3400 \mathrm{~m})$, a positive (negative) divergence corresponds to negative (positive) vertical velocity in this atmospheric layer. Equation (8) is basically the continuity equation for precipitation originally derived by Kessler (1995). The first term on the right represents the sedimentation (vertical advection) of rainwater, and the second term represents growth by condensation. Since cloud water $q_{c}$ 
TABLE 1. Synthetic description of the parameters in the model.

\begin{tabular}{cl}
\hline \hline Parameter & \multicolumn{1}{c}{ Description } \\
\hline$p_{0}$ & Condensation \\
$p_{1}$ & Evaporation \\
$p_{2}$ & Vertical velocity lag \\
$p_{3}$ & Outflow divergence \\
$p_{4}$ & Forward-backward outflow factor \\
$p_{5}$ & Mix-winds weight factor \\
\hline
\end{tabular}

is not considered in this model, the microphysical contributions to precipitation as a result of autoconversion of cloud to rain and the accretion of cloud water by existing rain are not accounted for. In addition to sedimentation and condensation, evaporation needs to be represented in the model to ensure a precipitation balance. Following Kessler (1995) the depletion of rain by evaporation can be represented as

$$
\frac{\partial q_{r}}{\partial t}=p_{1} q_{c} q_{r}^{0.65}
$$

where $p_{1}$ is the evaporation parameter (Table 1), and with a strong approximation $q_{c}$ has to be assumed constant $(=1)$ for being not represented in this context. For practical implementation, since only two vertical levels are considered, the parametric equations given above are applied to the upper (superscript 1) and lower (superscript 0) levels:

$$
\begin{aligned}
\left(q_{r}\right)_{t+1}^{1} & =\left(q_{r}\right)_{t}^{1}+p_{0} L^{p_{2}} w_{t} d t ; \quad L^{p_{2}} w_{t}=w_{t-p_{2}}, \\
\left(q_{r}\right)_{t+1}^{0} & =\left(q_{r}\right)_{t}^{0}+\frac{\left(q_{r}\right)_{t}^{1}-\left(q_{r}\right)_{t}^{0}}{\Delta z}\left(w+V_{t}\right) d t \\
\left(q_{r}\right)_{t}^{1} & >\left(q_{r}\right)_{t}^{0} \quad \text { and } \quad\left(w+V_{t}\right)<0, \quad \text { and } \\
\left(q_{r}\right)_{t+1}^{0,1} & =\left(q_{r}\right)_{t}^{0,1}+p_{1} q_{r}^{0.65} d t,
\end{aligned}
$$

where $t$ and $t+1$ indicate the current and next time step, respectively; and $d t$ is the forecast time interval. In Eq. (11) $L$ denotes the lag operator; that is, $L^{p_{2}}$ means lagging $w$ by a period $p_{2}$ (vertical velocity lag parameter). The lagged field is obtained by advecting backward or forward in time the divergence (vertical velocity) using the estimated motion vectors. This is a necessary and important feature of the model to compensate for the lack of three-dimensionality, in particular for squall lines with surface-based cold pools. In fact the regions of strong convergence near the surface, often associated with a gust front (Fig. 4), may move significantly away (often downwind) from the main storm core. This may result in a tilted warm inflow current turning into the updraft. In this case the storm enhancement or new cell development will take place downwind with respect to the observed low-level convergence.
The role of the lagged vertical velocity is therefore to heuristically contemplate the possible spatial displacement. After all, although the storm evolution is greatly dependent on the environmental shear profile (Rotunno et al. 1988), it is believed that the relative position and propagation of the near-surface convergence with respect to the precipitation core has the potential to provide valuable information to improve the very shortterm prediction of the overall system motion and morphology.

Rainwater vertical advection is applied only when there is a positive $q_{r}$ flux downward [Eq. (12)] in the current setting. Equation (12) may actually be applied irrespective of the gradient and vertical velocity signs, although this may imply negative $q_{r}$ values. Negative $q_{r}$ can be dealt with and provide a way to inhibit new convection in regions where persistent downdrafts have occurred. However, some preliminary tests showed that the adopted solution, exploiting the evaporation term to balance the overall rainwater budget, performed better.

The coefficients $p_{0}$ (condensation), $p_{1}$ (evaporation), and $p_{2}$ (lagged vertical velocity) are part of the parameters set to be determined adaptively through the optimization described below in section $4 \mathrm{~d}$.

\section{b. Outflow parameterization}

A downdraft generally develops within a thunderstorm when precipitation falls through an unsaturated layer and evaporation starts cooling the air. The combined effect of precipitation loading (drag of liquid water) and evaporative cooling can lead to the formation of a cold pool, which is associated with damaging winds at the surface. In fact the downdraft approaching the surface causes a divergent flow and a gust front (outflow boundary) propagates, separating the cooler air below the storm core from the environmental warmer air (Cotton et al. 2010). The speed of the advancing gust front relative to the ambient flow is found to be close to the speed of a density current and can be expressed in terms of the density difference between the surrounding air and the denser air within the cold pool (Wakimoto 1982). However, in this context the lack of any information about density (or pressure) prompts an attempt to represent the flow associated with the cold pool in terms of the vertical velocity and the evaporative cooling [Eq. (10)]. Because of the nonuniform surface winds and the three-dimensional flow organization, in particular the presence of a rear inflow jet in squall lines causing cold air to be drawn in on the rear side of the storm (Cotton et al. 2010), the divergent flow on the two-dimensional plane near the surface is in general not symmetric. To mimic this near-surface two-dimensional structure of the flow 


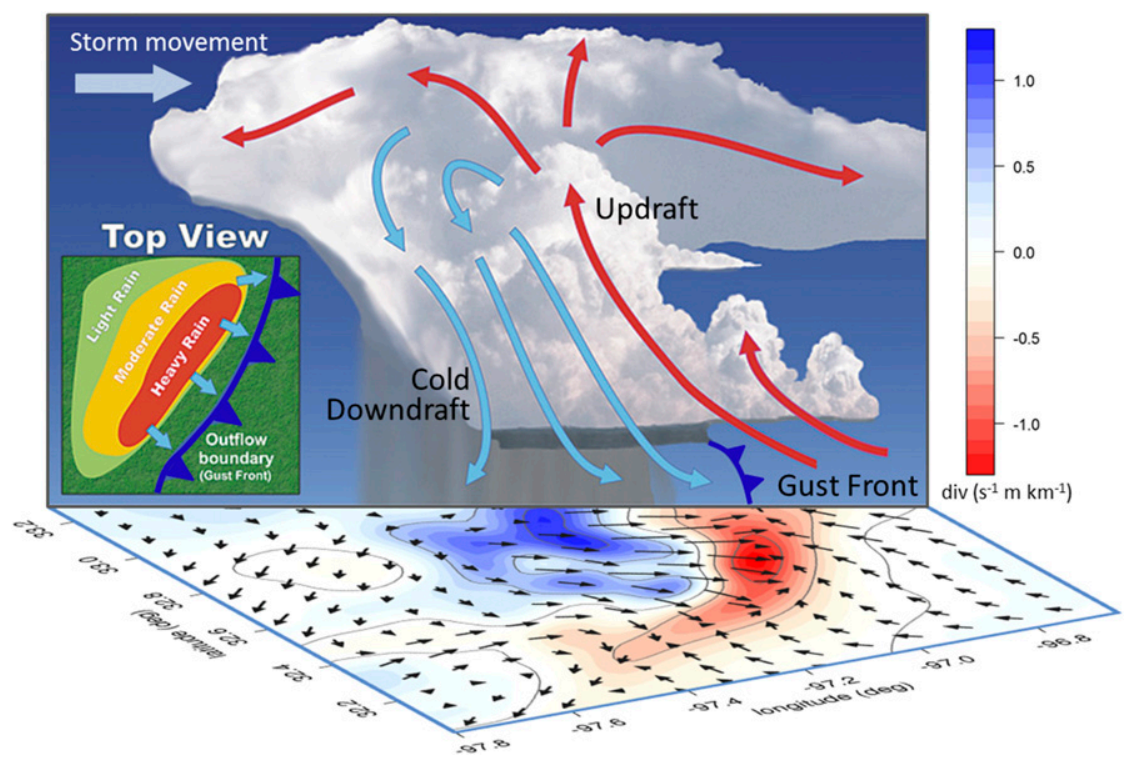

FIG. 4. Idealized diagram of a squall-line vertical structure showing updrafts, downdrafts, and a gust front. Precipitation forming in the tilted updraft falls into the downdraft. Beneath the cloud, the denser cool air of the downdraft spreads out along the ground. On the leading edge of the outflowing downdraft, a gust front may form, forcing the moist surface air to flow up into the cloud. In the lower horizontal plane oriented perpendicular to the diagram, a real VDRAS wind analysis from the case study discussed in section 5 (2021 UTC) is displayed with divergence (color). The diagram and the horizontal analysis are subjectively matched with the purpose of illustrating the relation between the low-level wind and the storm vertical development. From the retrieved wind field, the average storm motion has been subtracted in order to show the storm-relative winds. The diagram is adapted from the National Weather Service Online Weather School (http://www.weather.gov/jetstream/tstrmtypes).

originating in downdraft regions, a term defining the outflow strength is first introduced:

$$
\begin{aligned}
\mathrm{DIV}_{\text {out }} & =p_{3}\left(w+V_{t}\right) d E d t \\
d E & =\left[\left(q_{r}\right)_{t+1}^{0}\right]^{0.65}-\left[\left(q_{r}\right)_{t}^{0}\right]^{0.65}, \\
d E & >0 \quad \text { and } \quad\left(w+V_{t}\right)<0
\end{aligned}
$$

where $p_{3}$ represents the outflow divergence parameter (Table 1 ), and $d E$ is the incremental rainwater mass loss owing to evaporation between time steps $t$ and $t+1$. Equation (14) is based on the knowledge that the outflow produced at the surface is the consequence of both the negative vertical velocity (producing divergence) and cooling resulting from evaporation (producing a spreading density current). The initial wind analysis already implicitly includes the outflow term, while for the next predicted time steps the evolution of both the downdraft velocity and the rainwater mass needs to be considered.

From the divergence term associated with the downdraft [Eq. (14)], the corresponding irrotational flow $\left(\mathbf{U}_{\text {out }}\right)$ is estimated using an SOR technique and recalling Eqs. (5) and (6):

$$
\mathbf{U}_{\text {out }}=\nabla \chi ; \quad \nabla^{2} \chi=\delta,
$$

where $\chi$ is the velocity potential and the Dirichlet boundary condition (null velocity on the boundary) is assumed.

The combination of the mean wind and the rear inflow with the outflow causes the circulation within the cold pool to divert from the symmetric pattern arising from the SOR retrieval. In practice over time the cold pool tends to elongate in the direction of the mean wind Corfidi (2003), with segments of the gust front oriented parallel to the mean wind remaining quasi stationary, while segments perpendicular to the mean wind move faster downwind. This behavior can be portrayed considering the angle $\theta$ between the unit vector representing the motion of the wind field divergence (indicative of the gust front propagation) and the irrotational flow vector associated with the outflow [Eq. (15)]. A damp/ strengthen factor is defined based on the dot product between these two vectors as

$$
d=0.5[p 4 \cos (\theta)+1]
$$

and applied to the outflow vector: 

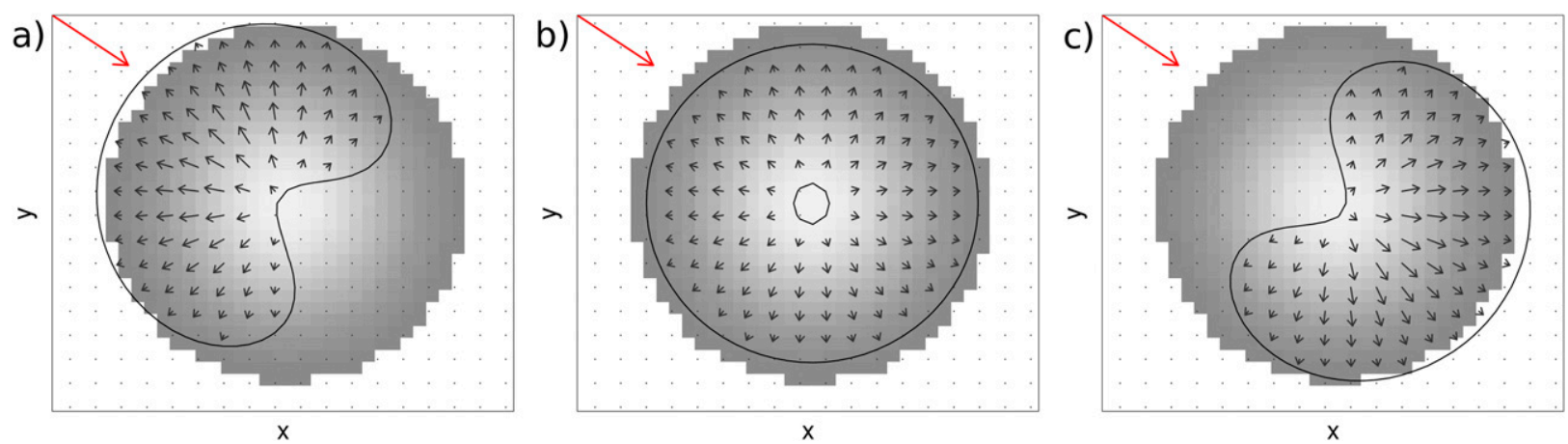

FIG. 5. Qualitative illustration of the flow representation within the cold pool on an arbitrary $x-y$ plane. Shown are the intensity of the divergence (gray shading), the storm-relative wind in the cold pool (black arrow), the direction of the divergence motion (red arrows), and the outflow boundary (solid contour). (a) Backward $\left(p_{4}=-1\right)$, (b) symmetric $\left(p_{4}=0\right)$, and (c) forward $\left(p_{4}=+1\right)$ propagation.

$$
\mathbf{V}_{\text {out }}^{\prime}=d \cdot \mathbf{U}_{\text {out }} \text {. }
$$

When the forward-backward outflow factor parameter $p_{4}$ is null $\left(p_{4}=0\right)$, the flow is unaffected except for a 0.5 scaling factor (Fig. 5b), while values of $p_{4}<0$ and $p_{4}>0$ are associated with backward and forward propagation, respectively (Figs. 5a,c, respectively). The resulting flow $\mathbf{V}_{\text {out }}^{\prime}$ is finally added to the low-level wind field:

$$
\mathbf{U}^{\prime}=\mathbf{U}+\mathbf{V}_{\text {out }}^{\prime}
$$

\section{c. Wind advection and mixing with rainwater motion vectors}

Vorticity and divergence are calculated from Eq. (18) and advected using the respective motion vector estimates. The wind field at time step $t+1\left(\mathbf{U}_{t+1}^{\prime}\right)$ is then calculated applying the SOR technique from the divergence and vorticity components, relying on the current time wind field $\left(\mathbf{U}_{t}^{\prime}\right)$ as the first guess. The updated low-level wind field is also used to adjust the rainwater motion vectors, based on the previously discussed assumption that the storm motion is influenced by the mean wind in the low to medium troposphere:

$$
\mathbf{U}_{q r}=\left(p_{5} \cdot \mathbf{U}^{\prime}+\mathbf{U}_{q r}\right) /\left(p_{5}+1\right) .
$$

The flow-related coefficients $p_{3}, p_{4}$, and $p_{5}$ complete the set of six parameters (Table 1 ) that need to be determined.

The general procedure is represented by the flow diagram in Fig. 6 and summarized in the following points:

1) The analysis (rainwater and wind) at the current $\left(t_{0}\right)$ and previous $\left(t_{0}-1\right)$ time steps are considered as input for the model:

(i) image registration is applied in order to align the rainwater fields at the two vertical levels (600 and $3400 \mathrm{~m}$ ), compensating for possible real (wind shear) or apparent (interscan delays) tilting of the storm; and

(ii) the intensity of the outflow, and the relative flow vectors, is estimated from the rainwater and divergence at time $t_{0}$ according to Eq. (14) (blue blocks in the flow diagram).

2) Optical flow estimation of the motion vectors is performed separately for the three fields: $q_{r}$, and the two derived components of the wind field, that is, $\delta$ and $\xi$.

3) Growth/decay terms are applied to the $q_{r}$ fields (lower and upper levels) according to Eqs. (11)-(13).

4) The fields $q_{r}, \delta$, and $\xi$ at $t_{0}$ are advected to time step $t_{0}+1$ using the respective motion vectors (point 2), relying on a backward advection scheme with bicubic interpolation.

5) From the updated $q_{r}$ and $\delta$ at time $t_{0}+1$, the new intensity of the outflow and relative flow vectors is also estimated.

6) The advected $\delta$ and $\xi$ are combined with the outflow from point 1(ii) (which is also advected using the motion vectors $\mathbf{U}_{\delta}$ ) to retrieve the updated low-level wind field at time step $t_{0}+1$.

7) The updated low-level wind field is mixed with the $q_{r}$ motion vectors at time $t_{0}$ to provide new displacement vectors to advect $q_{r}$ forward from $t_{0}+1$ to $t_{0}+2$.

For the successive time steps, points 3-7 are cyclically repeated, incrementing the time indexes. The time step used in the forecast is the same as the time interval between the pair of initial observations $t_{0}$ and $t_{0}-1(292 \mathrm{~s})$. Experiments using shorter time steps for the forecast resulted in small differences.

\section{d. Optimization of the model parameters}

To find the optimal set of parameters, the NelderMead (NM) downhill simplex method [Nelder and Mead (1965)] is adopted. The NM method belongs to 


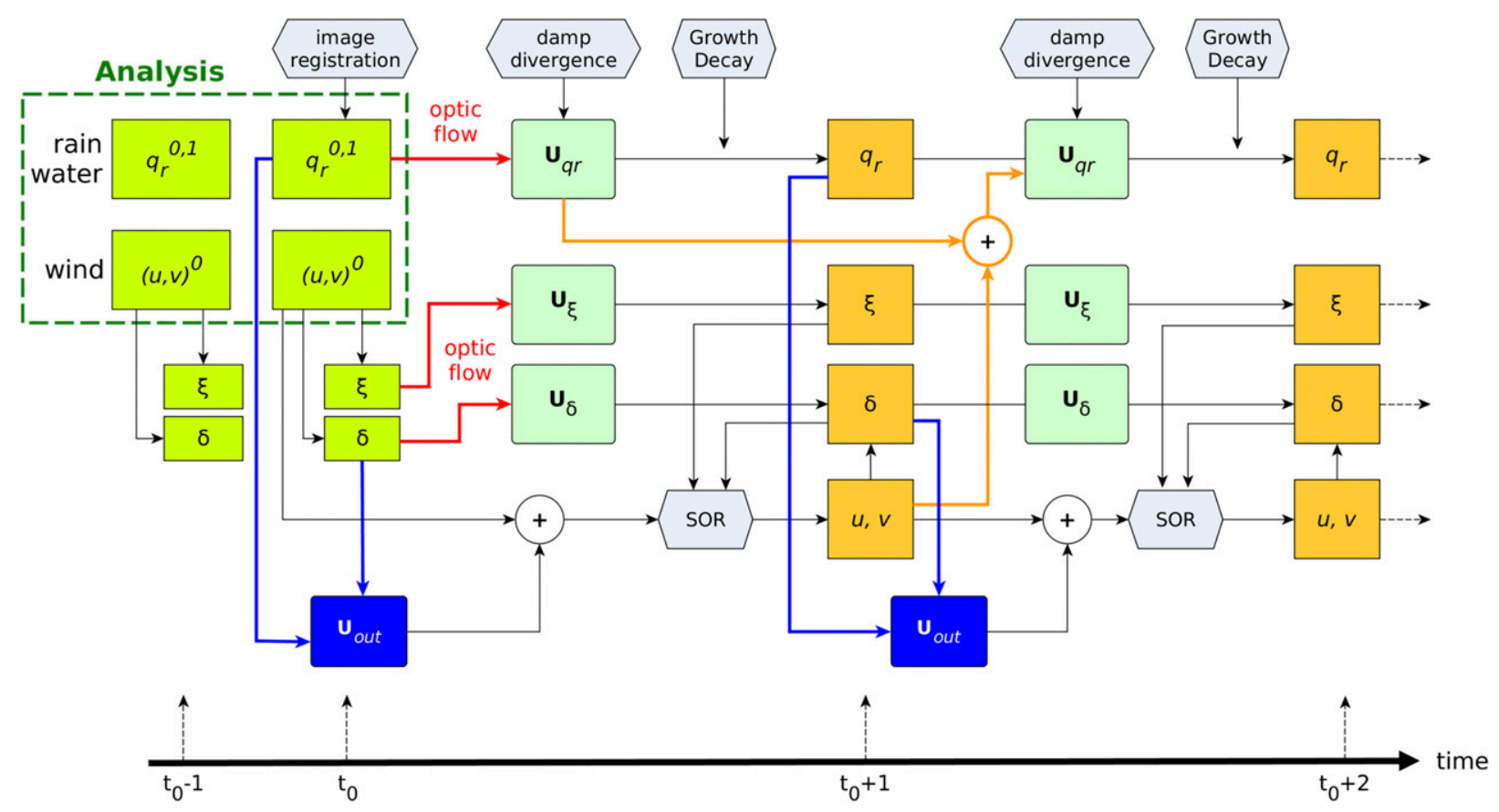

FIG. 6. Schematic flow diagram of the nowcasting model. The growth/decay process includes sedimentation, condensation, and evaporation (section 4a). The colors indicate observations/analysis (green background), the motion vectors (light green), the forecast fields (orange), and the outflow modeling (dark blue). The orange arrows represent the wind mixing (section $4 \mathrm{c}$ ). Superscripts 0 and 1 in the analysis fields refer to the lower $(600 \mathrm{~m})$ and upper $(3400 \mathrm{~m})$ levels, respectively.

the class of direct search methods and is suitable for multidimensional unconstrained optimization. The simple grounding idea and ease of implementation makes it a very popular method, used in a wide range of scientific applications. The NM method is not a true global optimization algorithm, although in practice it tends to work reasonably well for problems that do not have many local minima. The objective function to minimize in the current application is defined by the following sum of root-mean-square errors:

$$
\begin{gathered}
f=\frac{\left\langle\left(q_{r}^{\mathrm{obs}}-q_{r}^{\mathrm{fcst}}\right)^{2}\right\rangle^{0.5}}{\sigma_{q r}}+\frac{\left\langle\left(u^{\mathrm{obs}}-u^{\mathrm{fcst}}\right)^{2}\right\rangle^{0.5}}{\sigma_{u}}+\frac{\left\langle\left(v^{\mathrm{obs}}-v^{\mathrm{fcst}}\right)^{2}\right\rangle^{0.5}}{\sigma_{v}}, \\
\sigma_{q r}=0.2 \mathrm{~g} \mathrm{~kg}^{-1} ; \quad \sigma_{u}=3.0 \mathrm{~m} \mathrm{~s}^{-1} ; \quad \sigma_{v}=3.0 \mathrm{~m} \mathrm{~s}^{-1}
\end{gathered}
$$

where the average is calculated over the space-time validation domain. The normalization factors in the denominator are assumed constant. The spatial domain is a subdomain of the whole model domain (Fig. 8), to avoid boundary effects, while the temporal domain extends from the analysis time until a given forecast lead time (e.g., $60 \mathrm{~min}$ ).

\section{Results}

Considering the period between 1947 and 2100 UTC 12 May 2014, a total of 16 analyses (one every 292 s) are available. For each analysis a forecast up to $+58 \min$ (12 time steps with $d t=292 \mathrm{~s}$ ) is started, using the current and the lag- 1 fields for the optical flow estimation. The forecasted $q_{r}$ and low-level winds $\mathbf{U}$ are compared with the corresponding analyses for the estimation of the function $f$ in the iterative optimization procedure [Eq. (20)]. In the current implementation, all the parameters in the model (Table 1) are scaled during the forecast by a factor of 1 (forecast $+0 \mathrm{~min}$ ) linearly decreasing with lead time until zero (forecast + $120 \mathrm{~min}$ ). In fact, since the model greatly relies on extrapolation $\left(q_{r}\right.$ and winds) and the domain is limited (both the spatial domain and the variable space), the simple physical relations adopted [Eqs. (11)-(19)] will 

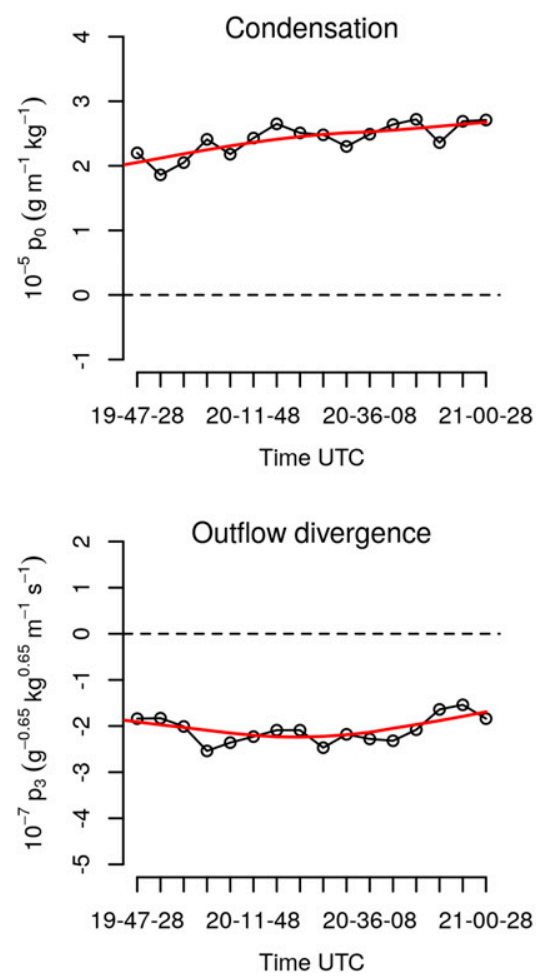
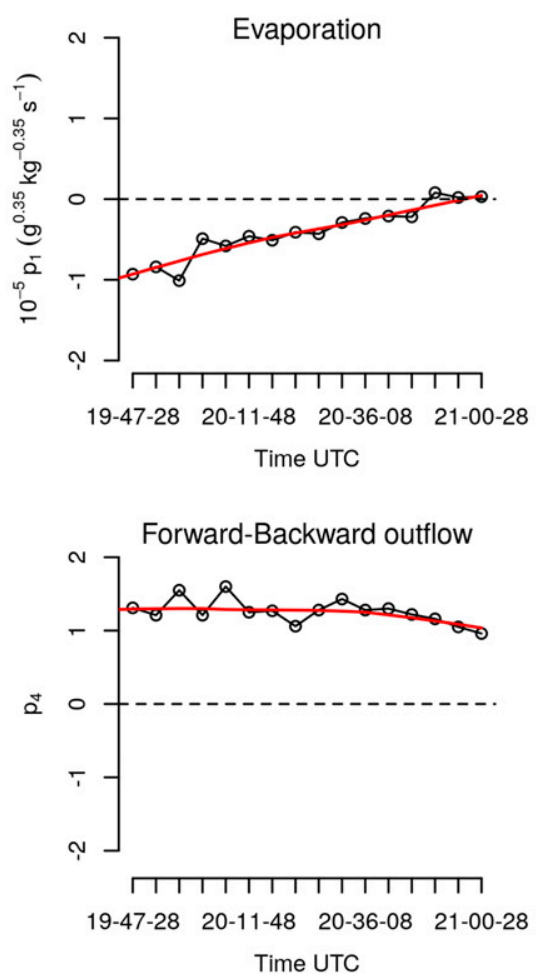
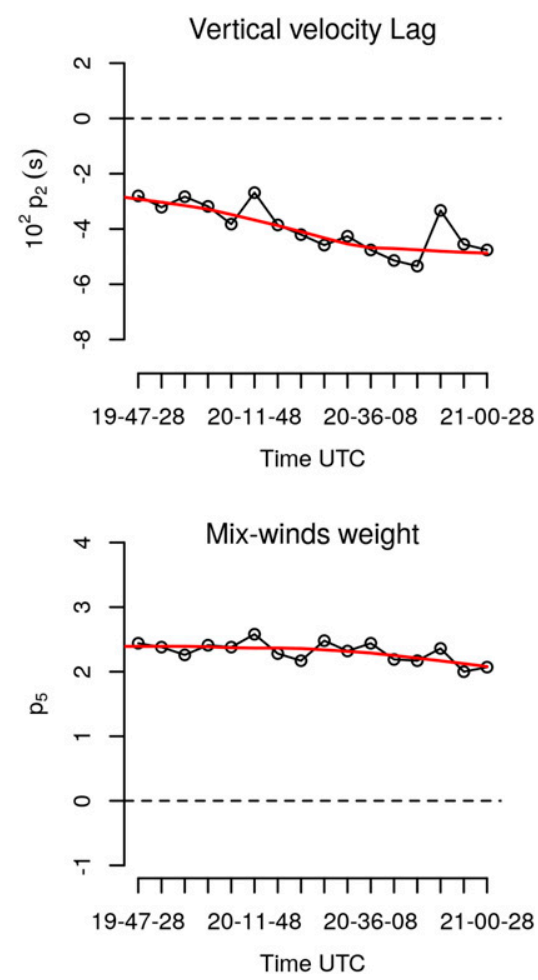

FIG. 7. Parameters obtained after NM optimization over 58-min forecasts between 1947 and 2100 UTC. A spline fit is superimposed (red) to highlight the trend of the parameters with time.

inherently lose their adequacy during the forecast. The decreasing scaling factor is then adopted to give more confidence to the physical relations during the first stages of the forecast while trying to keep the performance robust for longer lead times.

The validation is performed on the domain (represented by the rectangle in Fig. 8, $89 \times 94$ grid points), with a $2-\mathrm{km}$ grid resolution. The results of the current method are compared with the simple Horn-Schunck optical flow method described in section 3, based on $q_{r}$ motion vectors (called OF).

For each of the 16 analyses in the study period, the Nelder-Mead optimization is performed to determine the optimal set of model parameters. The optimization is performed without constraints, but the choice of the initial value of the parameters has an impact on the result, since Nelder-Mead is not a true global optimization algorithm and it may converge to different local minima depending on the initial setting. One way to overcome this limitation would be to perform an outer loop utilizing many initial simplicities in order to find the most suitable part of the parameter space with which to start. In this preliminary evaluation, the initial parameters are set to "reasonable" values based on the physical meaning of the processes involved $\left(p_{0}\right.$ and $p_{5}$ are expected to be positive, while $p_{1}, p_{2}$, and $p_{3}$ should assume negative values) and trial and error forecast runs with varying configurations. The adopted initial set of values is as follows: $p_{0}=+2.0 \times 10^{-5}, p_{1}=-0.5 \times 10^{-5}$, $p_{2}=-600 \mathrm{~s}, p_{3}=-1.5 \times 10^{-7}, p_{4}=+0.8$, and $p_{5}=+2.0$. During the optimization, a 58-min forecast is run iteratively until convergence is reached. On average a single optimization loop took about 18 iterations and 39 function evaluations. The resulting parameters are plotted in Fig. 7 for every independent forecast. It can be seen that, although the 16 optimizations at successive times are performed independently, ${ }^{1}$ the resulting parameters are substantially stationary or smoothly changing.

The parameter showing the most significant relative variation is $p_{1}$ (evaporation), passing from about $-1.0 \times$ $10^{-5}$ at 1947 UTC to $\sim 0$ around 2050 UTC. As discussed further later in this section, the evaporation term contributes little or nothing to the skill of the forecast when evaluated using the threat score, but it is useful for

\footnotetext{
${ }^{1}$ However, the initial analyses are not completely independent. In fact, for a given analysis time the VDRAS assimilation relies on the background forecast from the previous cycle. This contributes to guaranteeing physical consistency among successive analyses, and it can also reflect in the smooth evolution of the parameters resulting from the optimization.
} 

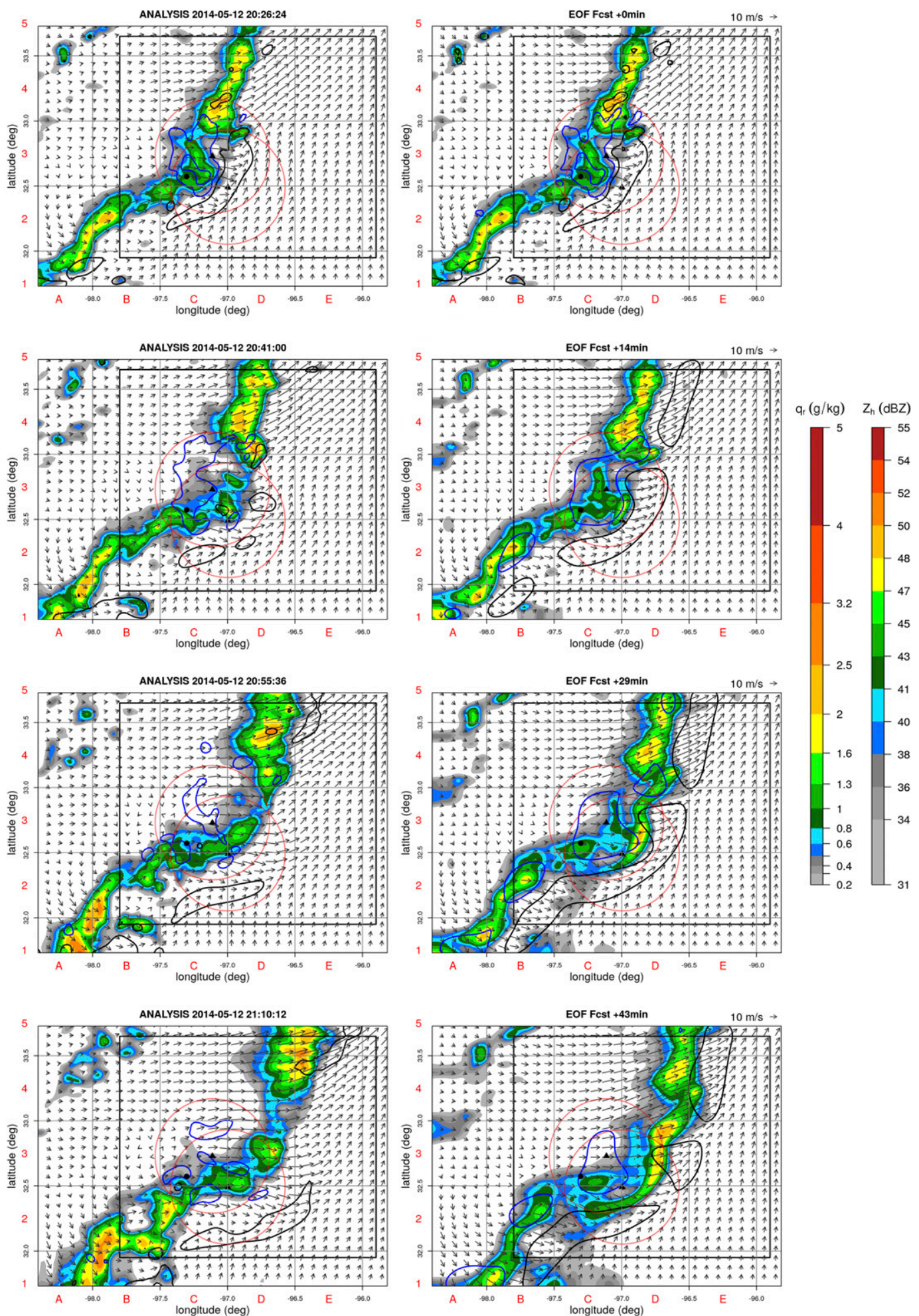

FIG. 8. (left) Analysis and (right) corresponding forecasts every $\sim 15 \mathrm{~min}$ of rainwater and low-level winds starting at 2026 UTC. The solid contours represent the 0.5 (blue) and $-0.5 \mathrm{~s}^{-1} \mathrm{~m} \mathrm{~km}^{-1}$ (black) divergence levels. The domains of the X-band radars (red circles) and the validation area (rectangle) are marked. The color palette for reflectivity $(\mathrm{dB} Z)$ is defined assuming a $Z\left(q_{r}\right)$ relation as in Sun and Crook (1997). 

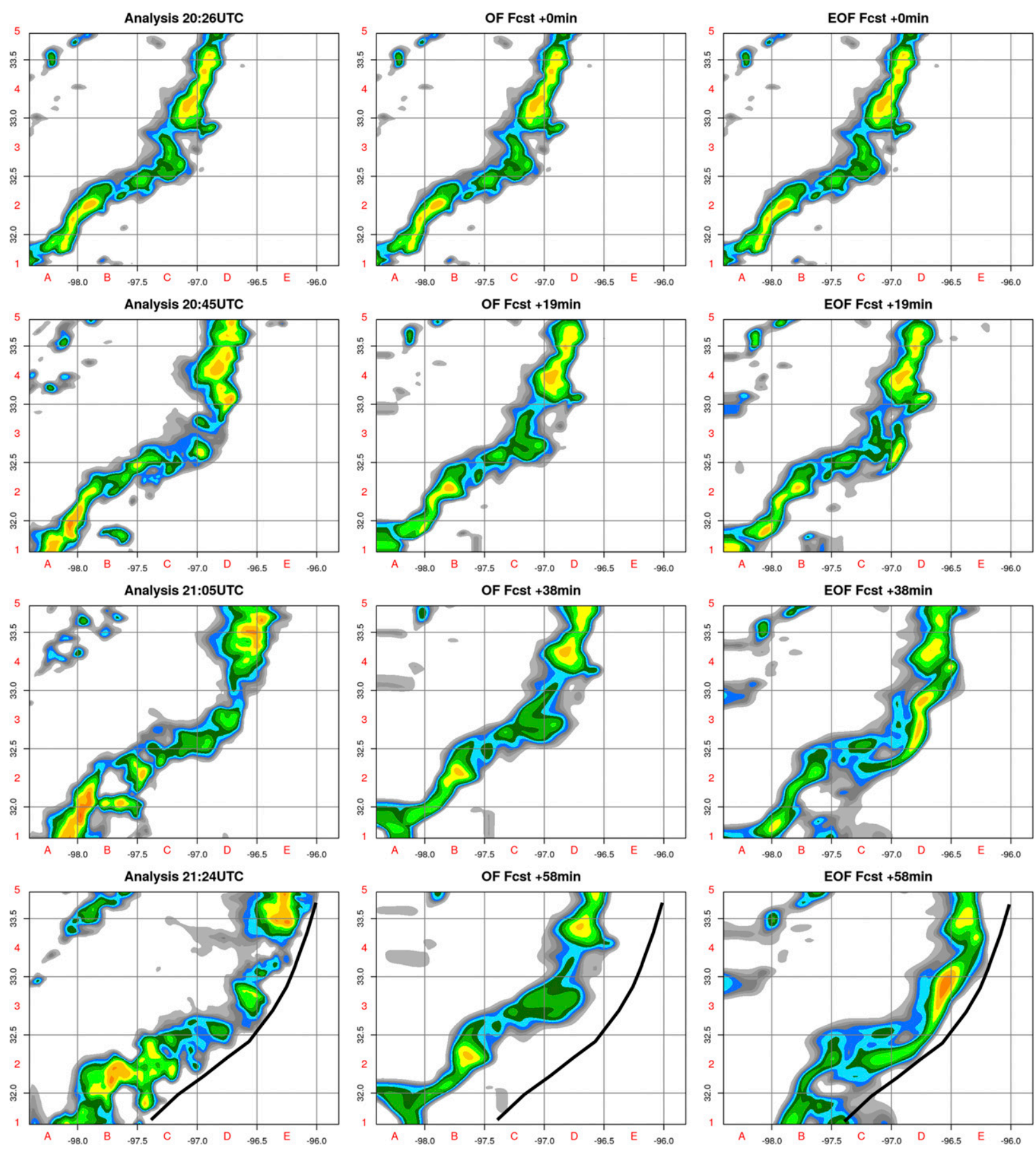

FIG. 9. (left) Analysis between 2026 and 2124 UTC, (center) corresponding forecasts every $\sim 20$ min using standard OF, and (right) parametric model forecast (EOF). The color palette is as in Fig. 8. For ease of comparison, the curved black line in the bottom row marks the approximate position of the storm frontal line from the analysis.

maintaining the average rainwater level close to the observations.

As an example, Fig. 8 shows the parametric model forecast starting at 2026 UTC, denoted enhanced optical flow (EOF). In this and the following analysis/forecast images, a coarse grid with $0.5^{\circ}$ latitude/longitude spacing is superimposed with letter/number coordinates in red to facilitate the comparison. The forecast for successive lead times are displayed in the right column, while the left column reports the corresponding analysis. In 

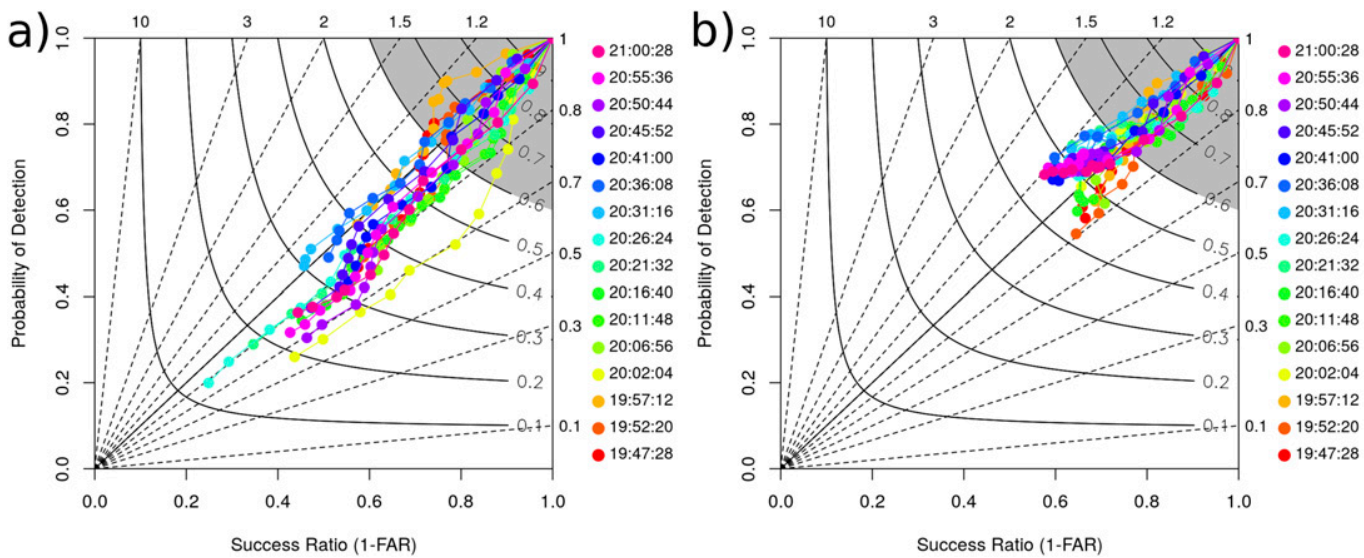

FIG. 10. Performance diagram for $q_{r}$ threshold of $0.4 \mathrm{~g} \mathrm{~kg}^{-1}$, corresponding to a reflectivity of $\sim 36 \mathrm{dBZ}$, for (a) OF and (b) EOF. The colors represent the forecasts starting at the analysis time in the legend, and the circles along a line indicate the successive forecast steps $(d t=292 \mathrm{~s})$. The final circle along each line corresponds to the +58 -min forecast.

addition to the rainwater (color palette), the analyzed and forecasted low-level $(600 \mathrm{~m})$ winds are displayed (arrows). In the +14-min forecast, a cell development associated with the outflow from the main storm is located fairly well just north of the Midlothian radar (lower-right triangle). This local enhancement associated with the gust front propagation is rather continuous in time in the forecast, while the analyses show a more pulsed behavior. In general the larger-scale morphology is depicted reasonably well until $\sim 1$-h lead time. In particular the model seems able to reproduce the increasingly faster movement of the northeastern portion of the storm and the broadening taking place southsouthwest of the three radars, where a cyclonic circulation developed. This is also evident in Fig. 9, where the parametric model forecast (EOF) of the rainwater is compared with the simple optical flow advection (OF). It is worth mentioning that this specific case is chosen for the sake of illustration, as result of the especially clear improvement of EOF over OF (refer also to Fig. 10, where the simple OF forecast starting at 2026 UTC shows the worst performance). The OF forecast tends to greatly underestimate the westward motion of the northeastern part of the storm, which appears to be caused by a combination of autopropagation (Cotton et al. 2010) and the stronger winds flowing into the region. In fact at a lead time of $+58 \mathrm{~min}$, the grid points $\mathrm{E} 4$ and E5 are still empty for OF, while the EOF forecast is in better agreement with the truth. Another relevant difference is in grid point $\mathrm{C} 2$. The southwestern part of the storm has a marked elongated shape at the analysis time, and the simple optical flow method tends to simply displace this pattern forward. The slower velocity with respect to the rest of the storm is well captured by both techniques, as demonstrated by the correct position of the rear boundary of the storm. However, the interaction of the southerly flow with the advancing storm is determining a broadening and intensification in this region that cannot be reproduced by a simple motion vectors advection. On the other hand, the parametric model is triggering new convection in this region. Although there are significant differences in magnitude and small-scale organization with respect to the actual analysis, the general agreement of the large-scale pattern appears valuable for nowcasting applications. From a qualitative perspective, the forecasted wind fields in Fig. 8 appear reliable over much of the domain until an approximately 30-min lead time, with a reasonable location of the main convergence regions. For larger lead times however, the actual gust front located over the overlapping region between the two X-band radars at 2026 UTC propagates faster than depicted in the forecast. At 2110 UTC the gust front in the analysis extends from $\mathrm{B} 1$ to $\mathrm{E} 2$, while in the forecast the gust front is located significantly behind (B1-D3).

For a quantitative evaluation of the proposed method, the well-known summary measures probability of detection (POD), threat score (TS), false alarm ratio (FAR), and bias are considered. Figure 10 shows the performance diagram (Roebber 2009), which allows for visualizing multiple measures of forecast quality on the same diagram. For good forecasts, POD, the success ratio (1 - FAR), bias, and TS approach unity, such that a perfect forecast lies in the upper-right corner of the diagram. The gray area is added to visually represent the region with threat score $>0.6$ in the upper-right portion of the diagram, which normally represents a good forecast. The left panel in Fig. 10 shows the performance of the simple optical flow (OF). The threat score decreases from 1 ( +0 -min lead time) to $0.2-0.3$ 

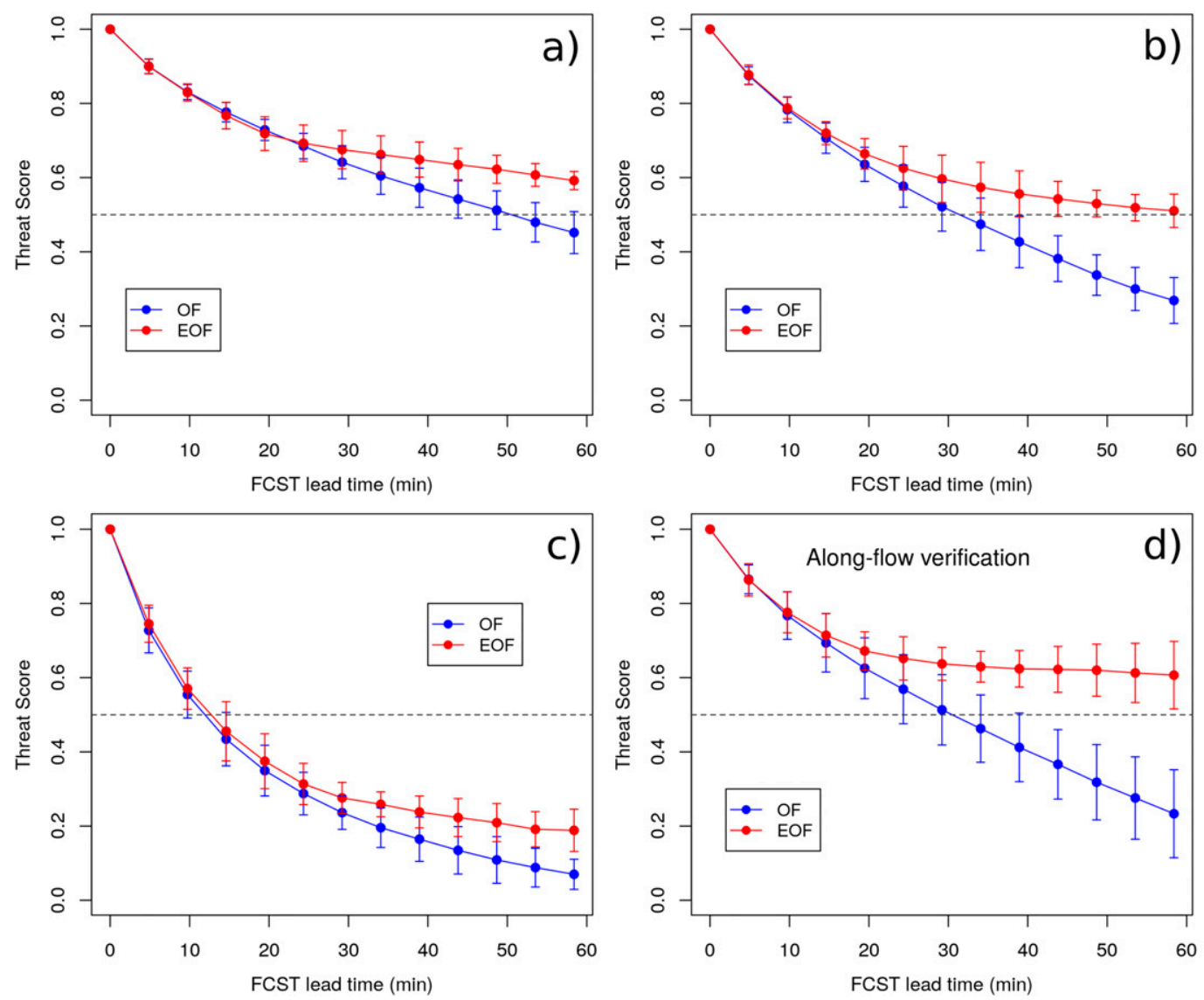

FIG. 11. Average TS (1947-2100 UTC) plotted for increasing forecast lead time and for three different $q_{r}$ thresholds corresponding to (a) 26, (b) 36, and (c) $\sim 43 \mathrm{dBZ}$. (d) TS is calculated for the same threshold as in (b), but "along the flow," i.e., over grid points originating from regions in the analysis where Doppler observations from at least two radars were available. This moving subregion extends over $\sim 20 \%-25 \%$ of the whole validation domain.

(+58-min lead time) for most of the forecasts. As previously anticipated, the forecast starting at 2026 UTC has the worst performance with a TS reaching almost 0.1 , while the 2002 UTC forecast shows a significant bias (0.6) after about 30 min into the forecast. In the right panel, the same diagram for the parametric model (EOF) depicts a significantly better performance, with a TS never reaching below 0.4 for the longest forecast. The bias is also very close to 1 for the first $30 \mathrm{~min}$ of the forecast and within the 0.8-1.2 range afterward.

The average TS over the different forecast runs is also summarized in Fig. 11, which reports the results for the EOF together with the corresponding performance of OF. Figures $11 \mathrm{a}-\mathrm{c}$ are for three different thresholds on $q_{r}$, namely, $0.1,0.4$, and $1.0 \mathrm{~g} \mathrm{~kg}^{-1}$, respectively, corresponding to a reflectivity of approximately 26,36 , and $43 \mathrm{~dB} Z$, respectively. The OF and EOF forecasts show similar skills for about 20-25 min, after which the EOF performs better. In particular for the $0.4 \mathrm{~g} \mathrm{~kg}^{-1}$ threshold, the TS for the EOF forecast lowers to 0.5 about
20 min later than for the OF forecast. For the other thresholds, a similar improvement is also observed for the larger lead times. This kind of performance would reflect in a sizeable impact in terms of advanced warning for real-time applications. The results displayed in Fig. 11d are for the same threshold as in Fig. 11b, that is, $0.4 \mathrm{~g} \mathrm{~kg}^{-1}$, but considering a validation area moving along the $q_{r}$ flow. The verification is performed on a portion of the whole validation domain, determined by the grid points that track back (using the model motion vectors) to the region in the analysis where collocated Doppler observations from at least two radars were available. In this way it is possible to size more specifically the impact of multiple Doppler observations on the forecast. It is in fact beneficial for the VDRAS assimilation to ingest Doppler observations from different radars, allowing for accurate retrieval of the twodimensional wind field. The density of the Doppler observations is advected similarly to $q_{r}$ and is used to define the along-flow validation domain for every forecast lead 


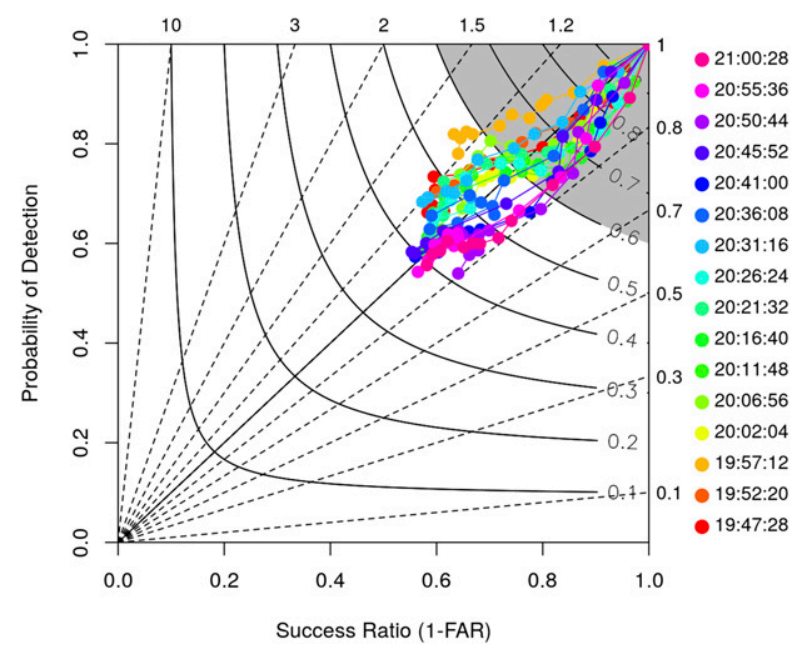

FIG. 12. As in Fig. 10, but using fixed parameters in the model.

time. The result in Fig. 11d shows an increased improvement upon the standard OF forecast (which did not changed substantially), corroborating the idea that a good wind analysis from multiple radars is crucial for the quality of the nowcasting.

The results illustrated so far represent the maximum achievable performance of the model for the given case study, since the results are optimized for each individual forecast using the future analyses. In a real-time application this is of course not possible, so the variability of the model parameters will have to be further analyzed using a comprehensive dataset to assess their validity for a wider range of meteorological situations. However, for this specific event, it is evident from Fig. 7 that most of the parameters do not show important variations during the event considered. As previously noted, this is likely ascribable to the specific VDRAS assimilation technique, ensuring the proper physical consistency in space and time and among the model variables. The observed slow time change of the parameters is encouraging for a hypothetical real-time application, when one cannot perform the optimization using future observations. In real time it may be possible to evaluate the set of parameters on the previous 40-60 min and use it for the current forecast.

To show the impact of neglecting the parameters' variation during the event, the performance using fixed parameters is evaluated. The parameters are simply set from the arithmetic average of the values resulting from the optimization (Fig. 7), that is, $p_{0}=+2.4 \times 10^{-5}$, $p_{1}=-0.5 \times 10^{-5}, p_{2}=-394 \mathrm{~s}, p_{3}=-2.0 \times 10^{-7}$, $p_{4}=+1.3$, and $p_{5}=+2.3$. The performance diagram corresponding to the forecasts with fixed parameters is presented in Fig. 12. Not surprisingly given the limited variations of the optimized parameters, these summary measures indicate a performance quite similar to the reference in Fig. 10, except for a more pronounced bias earlier in the forecast for some specific runs. For example, the 1957 UTC (light orange) forecast presents a positive bias, which is attributable to the lower evaporation coefficient $p_{1}\left(+0.5 \times 10^{-5}\right)$ with respect to the optimized value $\left(+1.0 \times 10^{-5}\right)$.

For the wind verification, no other nowcasting reference is available, so the parametric model results are compared with simple persistence, that is, assuming the $t_{0}$ analysis wind does not change during the forecast. Figures 13a,b show the average root-mean-square error (RMSE) for the zonal $(u)$ and meridional $(v)$ wind components, respectively, for increasing forecast lead times. These are the same errors used in Eq. (20) (second and third terms, respectively) for the optimization of the model parameters, so the clear improvement upon persistence is anticipated. The performance using fixed parameters (orange lines) is very similar, as the parameters directly affecting the wind forecast $\left(p_{3}\right.$ and $p_{4}$ ) are relatively constant during the event (Fig. 7).

The improvement of the wind components' $(u, v)$ forecast accuracy also reflects on the wind direction, and to a lesser degree on the wind velocity (Figs. 13c,d). In percentage the relative improvement for the wind direction is over $30 \%$ at an approximately 60 -min lead time, while only $10 \%-15 \%$ for the wind velocity.

To assess the relative impact of the individual terms in the parametric model, a number of experiments are performed by selectively suppressing some of the processes. This is realized by setting to zero the parameter(s) controlling a given process and rerunning the optimization procedure on a reduced number of parameters. In this way, although the subset of the remaining parameters may converge to different values with respect to the full model configuration, the summary statistical measures allow for evaluating the relevance to the nowcasting of the individual components of the model. Figure 14 reports the results for the performance of rainwater nowcasting (left panel) and wind direction (right panel). The impact on the wind velocity is not considered because the improvement obtained with the full model (Fig. 13) is quite small (the difference between the RMSE of persistence and EOF is within $\pm \sigma$ ), and the even smaller differences achievable with the partial model cannot be reliably evaluated.

Instead of reporting the TS as in Fig. 11 for $q_{r}$, in the left panel of Fig. 14 the difference between the EOF and OF threat scores for the $0.4 \mathrm{~g} \mathrm{~kg}^{-1}$ threshold (i.e., the improvement upon simple optical flow) is displayed to better appreciate the smaller differences. The solid red line with circles is the difference between the threat scores of EOF and OF as in Fig. 11b and represents the 

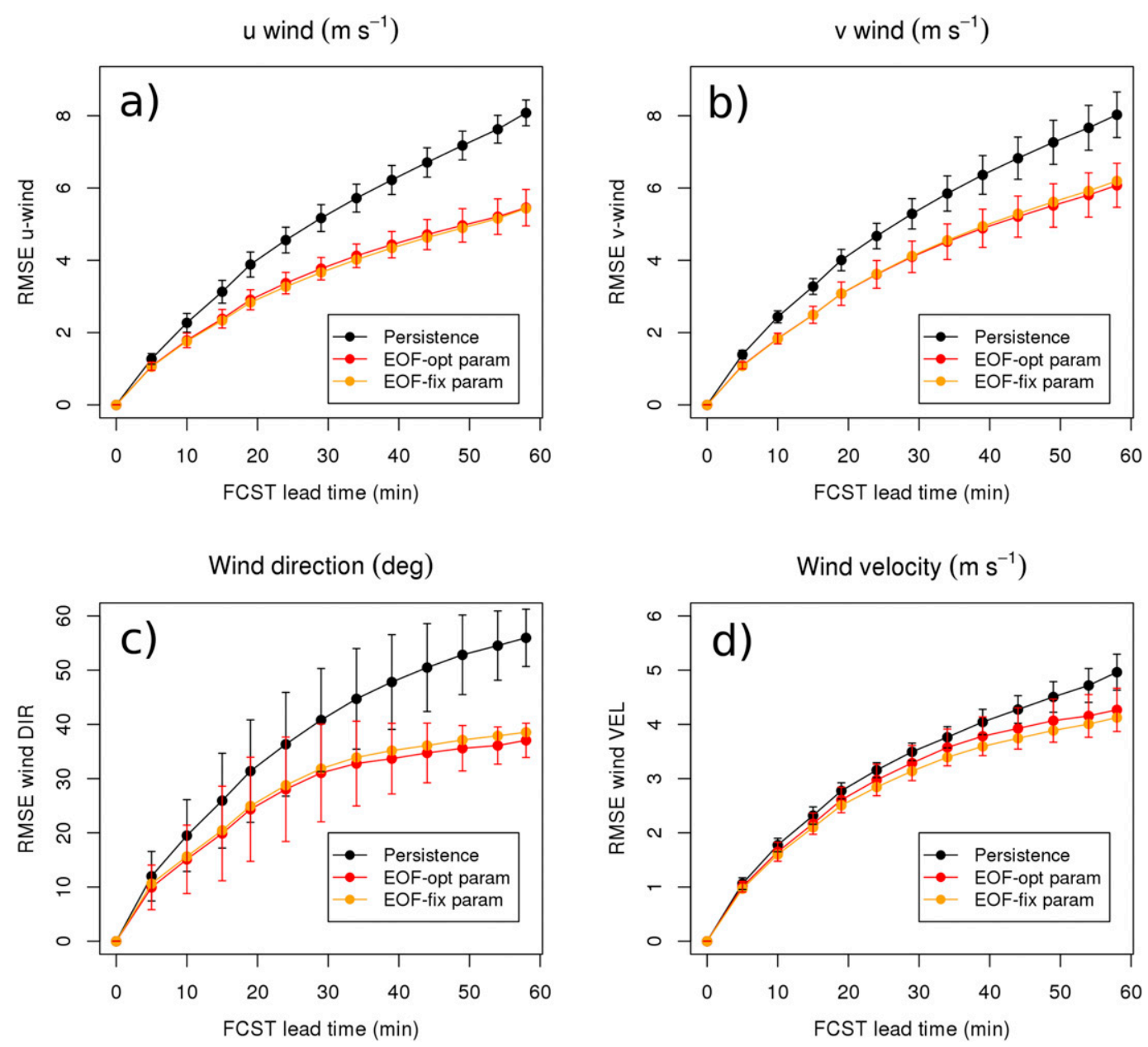

FIG. 13. Average RMSE for (a) $u$ and (b) $v$ wind components, and for wind (c) direction and (d) intensity for increasing forecast lead time. The error associated with the wind prediction assuming persistence (black), and the error for the extrapolation model forecast with optimized (red) and fixed (orange) parameters. The error bars, corresponding to $\pm \sigma$, are omitted for clarity.

reference performance with the full model: the lower the TS difference for the other experiments, the more relevant the process removed from the forecast.

It is clear that the removal of the evaporation process alone (dashed pink line) has a negligible impact on the threat score, as previously anticipated. The bias (not shown) remains very close to 1 because $p_{0}$, which is close to zero during the first part of the event, compensates for the lack of evaporation. On the other hand, removing the condensation process (alone or together with the evaporation, solid and dashed blue lines, respectively) has a sizeable effect on the overall performance of the rainwater forecast. For the wind forecast (right panel in Fig. 14), evaporation and condensation have little impact. This is somehow expected, since these processes directly affect the $q_{r}$ distribution and only secondarily the wind field through the ouflow parameterization. This last process (outflow) is indeed the most relevant for the wind field, since it explicitly influences the flow around the cold pool region below the storm core. Less obviously the outflow modeling is also very important for the rainwater nowcasting. This highlights that a proper forecast of the gust front propagation is essential to ascertain the location where the storm enhancement or new convection will occur. In the proposed approach, this aim is pursued through 1) the forecast of the divergence field using the optical flow technique and 2) the parameterization (parameters $p_{3}$ and $p_{4}$ ) of the outflow below the precipitation cores. The last parameter considered in the model, the mix-winds weighting factor $p_{5}$, has also a relevant impact on the rainwater forecasting skill. Compared to the outflow modeling, the update of the rainwater motion vectors using the low-level winds seems to have a larger impact earlier in the forecast. 

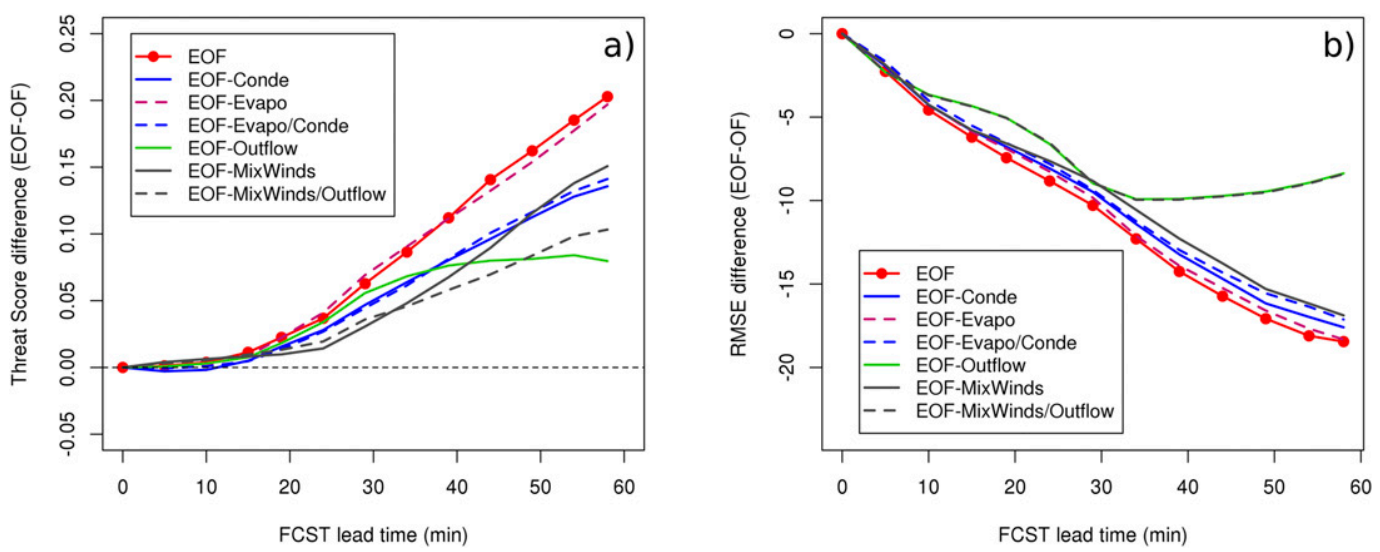

FIG. 14. Performance of the (a) rainwater and (b) wind direction forecast runs suppressing selected processes. The reference performance of the full model as in Fig. 11b is indicated relative to the OF forecast (red line). The forecasts without given processes (the name in the legend indicates the process suppressed) are indicated.

In fact the solid gray line in Fig. 14 starts deviating from the red line around a 20 -min lead time and then remains almost parallel to the reference performance. On the other hand, the forecast without the outflow parameterization becomes increasingly worse than the reference after a 30-min lead time. The solid gray (mix winds) and green (outflow) lines intersect around a 40-min lead time. This indicates that the outflow representation has indeed the longer-lasting effect on the quality of the forecast.

\section{Conclusions}

The basic idea behind the nowcasting approach discussed in this work is to extend the Lagrangian persistence concept, which is traditionally applied to reflectivity only (or related quantities, such as the rainfall rate, rainwater, etc.), to the components of the wind field, namely, divergence and vorticity. This clearly presumes the availability of a reliable wind analysis consistent with the radar observations in terms of coverage, and spatial and temporal resolution. In addition, some basic interactions between the wind and the rainwater advection have to be represented in the nowcasting model to allow the forecasted variables to evolve coherently, at least for short lead times.

The motivation for this work originates in part from the frustration of not being able in many instances to fully exploit the large amount of information provided by a Doppler weather radar. In the large majority of radar nowcasting applications nowadays, reflectivity is still the only observation being contemplated. While methods including dual-polarization variables have been proposed to improve the diagnosis and forecast of rainfall (Ruzanski and Chandrasekar 2012), the use of the Doppler information is in general largely unexploited.

The reasons are partly residing in the objective technical and conceptual difficulties, in particular when only measurements of a single Doppler radar are available. In addition to the advantages for the detection and quantitative estimation of precipitation, a dense networked radar system like the Dallas-Fort Worth test bed represents a unique tool for accurately sampling the winds near the ground. Overlapping coverage allows each point in the three-dimensional network domain to be simultaneously viewed by two or more radars, allowing for multiple-Doppler wind vector retrievals. Several geometric techniques for multiple-Doppler retrieval exist and can provide wind retrievals for various applications. However, in order to properly retain the physical consistency between the winds and the precipitation fields, the use of a numerical model appears fundamental.

As a matter of fact, the Doppler radar observations have been traditionally exploited in numerical models, being "naturally" prone to being ingested into data assimilation systems. The Doppler wind observations have in fact notable advantages. Radar wind measurements are immune to biases, while reflectivity is prone to calibration errors. In addition the wind is defined continuously all over the model domain (unlike rainwater, which is subject to the "on-off" switches in the warm rain process), facilitating the assimilation of the radial wind component.

The four-dimensional assimilation approach realized in VDRAS is specifically devised for the assimilation of weather radar data and is able to largely retain the observation component in the final analysis. However, while NWP data assimilation systems can provide fairly accurate and complete analyses, the model forecasts are still 
beaten by extrapolation techniques for very short-term prediction. It appears then reasonable to attempt taking the better of the two approaches, that is, the robustness and consistency of data assimilation for the analysis and the simplicity of extrapolation techniques for the subsequent nowcast. The method illustrated in this work represents a preliminary attempt to realize this idea. Despite a certain naivety of the basic model construction, the preliminary results of a single case study indicated a positive impact on the nowcast accuracy. However, more events encompassing a wide range of meteorological conditions will need to be evaluated to assess the actual potential of this technique, in comparison with standard extrapolation methods.

One peculiar aspect of this technique is the use of a parametric model, with parameters adaptable to the specific meteorological situation. For this preliminary application, the parameters have been determined a posteriori using an optimization method (Nelder-Mead), based on the deviation of the forecast from the corresponding analysis. However, it seems possible to devise a real-time application where the parameters are evolving based on the past observations. The analysis of more events will allow the estimation of reasonable ranges for the model parameters. Analogously to other probabilistic methods (Bowler et al. 2006), an ensemble forecast approach may be foreseen as a future development. In such probabilistic implementation, the uncertainty would not be limited to the initial conditions. Also, the parameter uncertainty should be represented using several model runs with varying parameter values.

For the case study illustrated, the achieved better performance of the EOF parametric model compared to the standard optical flow method is ultimately ascribable to the inclusion of the Doppler radar wind observations in the VDARS analysis, which allows for reliably representing the wind field around and ahead of the storm. This favors the representation of the condensation process leading to local storm enhancement, and the evolution of the wind field in the outflow region. On the other hand, the prediction of the local dissipation appears more difficult, and the simple evaporation process currently included did not contributed to the forecast skill.

In terms of computational requirements, this method is more demanding compared to extrapolation techniques based on optical flow techniques. Figure 15 shows a comparison between the wall-clock run times (running on a single-processor Intel $i 7$ at $2.67 \mathrm{GHz}$ ) for the different techniques during the case study considered. From bottom to top the time required to complete a $1-\mathrm{h}$ forecast goes from on the order of $1 \mathrm{~s}$ or less for OF to about 1-2 min for the parametric model, including optimization of the parameters (EOF-opt param). When using fixed

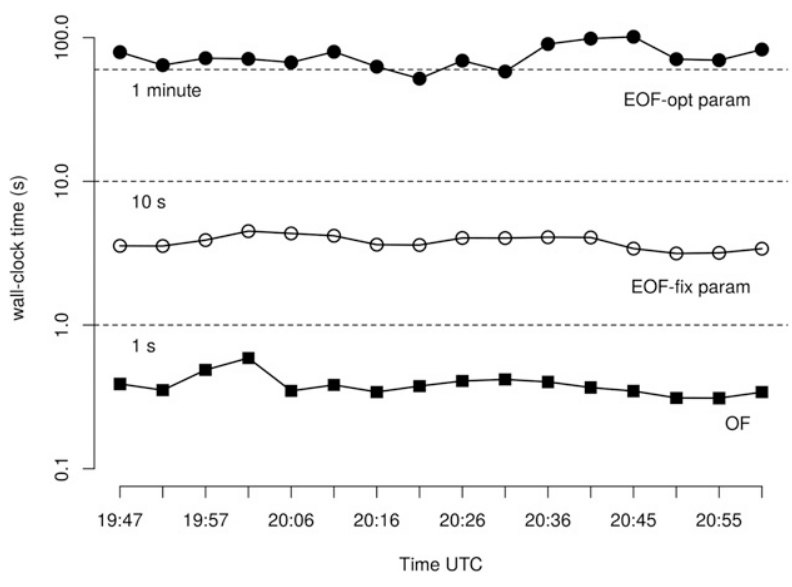

FIG. 15. Wall-clock execution times (s) for different nowcasting methods. Parametric model with optimized coefficients (filled circles), parametric model with fixed coefficients (open circles), and OF (filled squares). The $y$ axis is logarithmic.

parameters (EOF-fix param), the run time is about $5 \mathrm{~s}$. The computational complexity is on the order of $O\left(N_{\text {tot }}\right)$, so doubling the spatial resolution would result in about 4 times longer run times. For the parametric model method, however, the time required to elaborate the wind analysis (VDRAS) has to be added. The message passing interface (MPI) version of VDRAS was used in this study, running on a parallel computer using 16 processors. For the settings adopted in this experiment, the time required to complete an analysis with a 3 -min assimilation window is about $5 \mathrm{~min}$. Given the focus on the low-level wind analysis, it seems plausible to further decrease the needed run time, adopting a modified configuration with fewer vertical levels. These overall computational time requirements appear compatible with the possible real-time implementation.

Acknowledgments. This research is supported by the National Science Foundation Hazards SEES program (1331572). The meteorological analyses in this work were produced using the VDRAS package through collaboration with Juanzhen Sun of the National Center for Atmospheric Research. We are grateful to Juanzhen for her guidance and support in running the data assimilation experiments.

\section{REFERENCES}

Ballard, S. P., Z. Li, D. Simonin, and J.-F. Caron, 2016: Performance of 4D-Var NWP-based nowcasting of precipitation at the Met Office for summer 2012. Quart. J. Roy. Meteor. Soc., 142, 472-487, https://doi.org/10.1002/qj.2665.

Bechini, R., and V. Chandrasekar, 2015: A semi-supervised robust hydrometeor classification method for dual-polarization radar 
applications. J. Atmos. Oceanic Technol., 32, 22-47, https:// doi.org/10.1175/JTECH-D-14-00097.1.

- _ - — , and J. Sun, 2015: Four-dimensional variational data assimilation of high resolution X-band radar observations over the Dallas-Fort Worth metroplex. IGARSS 2015: Remote Sensing; Understanding the Earth for a Safer World, IEEE, 3953-3956, https://doi.org/10.1109/ IGARSS.2015.7326690.

Bowler, N. E., C. E. Pierce, and A. W. Seed, 2006: STEPS: A probabilistic precipitation forecasting scheme which merges an extrapolation nowcast with downscaled NWP. Quart. J. Roy. Meteor. Soc., 132, 2127-2155, https://doi.org/10.1256/ qj. 04.100 .

Bringi, V. N., and V. Chandrasekar, 2001: Polarimetric Doppler Weather Radar: Principles and Applications. Cambridge University Press, 648 pp., https://doi.org/10.1017/ CBO9780511541094.

Chornoboy, M. A., A. M. Matlin, and J. P. Morgan, 1994: Automated storm tracking for terminal air traffic control. MIT Lincoln Lab. J., 7, 427-448.

Cifelli, R., V. Chandrasekar, S. Lim, P. C. Kennedy, Y. Wang, and S. A. Rutledge, 2011: A new dual-polarization radar rainfall algorithm: Application in Colorado precipitation events. J. Atmos. Oceanic Technol., 28, 352-364, https://doi.org/ 10.1175/2010JTECHA1488.1.

Corfidi, S., 2003: Cold pools and MCS propagation: Forecasting the motion of downwind-developing MSCs. Wea. Forecasting, 18, 997-1017, https://doi.org/10.1175/1520-0434(2003)018<0997: CPAMPF $>2.0 . \mathrm{CO} ; 2$

Corpetti, T., E. Memin, and P. Perez, 2002: Dense estimation of fluid flows. IEEE Trans. Pattern Anal. Mach. Intell., 24 365-380, https://doi.org/10.1109/34.990137.

Cotton, W. R., G. Bryan, and S. C. van den Heever, 2010: Storm and Cloud Dynamics. International Geophysics Series, Vol. 99, Academic Press, 820 pp.

Davies, J. M., and R. H. Johns, 1993: Some wind and instability parameters associated with strong and violent tornadoes: 1 . Wind shear and helicity. The Tornado: Its Structure, Dynamics, Prediction, and Hazards, Geophys. Monogr., Vol. 79, Amer. Geophys. Union, 573-582, https://doi.org/10.1029/ GM079p0573.

Dérian, P., C. F. Mauzey, and S. D. Mayor, 2015: Wavelet-based optical flow for two-component wind field estimation from single aerosol lidar data. J. Atmos. Oceanic Technol., 32, 1759-1778, https://doi.org/10.1175/JTECH-D-15-0010.1.

Fitzpatrick, J. M., 1988: The existence of geometrical densityimage transformations corresponding to object motion. Comput. Vision Graphics Image Process., 44, 155-174, https:// doi.org/10.1016/S0734-189X(88)80003-3.

Germann, U., and I. Zawadzki, 2002: Scale-dependence of the predictability of precipitation from continental radar images. Part I: Description of the methodology. Mon. Wea. Rev., 130, 2859-2873, https://doi.org/10.1175/1520-0493(2002)130<2859: $\mathrm{SDOTPO}>2.0 . \mathrm{CO} ; 2$.

Hamada, M., P. Drian, C. F. Mauzey, and S. D. Mayor, 2016: Optimization of the cross-correlation algorithm for two-component wind field estimation from single aerosol lidar data and comparison with Doppler lidar. J. Atmos. Oceanic Technol., 33 81-101, https://doi.org/10.1175/JTECH-D-15-0009.1.

Haussecker, H. W., and D. J. Fleet, 2001: Computing optical flow with physical models of brightness variation. IEEE Trans. Pattern Anal. Mach. Intell., 23, 661-673, https://doi.org/ 10.1109/34.927465.
Heas, P., and E. Memin, 2008: Three-dimensional motion estimation of atmospheric layers from image sequences. IEEE Trans. Geosci. Remote Sens., 46, 2385-2396, https://doi.org/10.1109/ TGRS.2008.918167.

, — - N. Papadakis, and A. Szantai, 2007: Layered estimation of atmospheric mesoscale dynamics from satellite imagery. IEEE Trans. Geosci. Remote Sens., 45, 4087-4104, https:// doi.org/10.1109/TGRS.2007.906156.

Horn, B. K. P., and B. G. Schunck, 1981: Determining optical flow. Artif. Intell., 17, 185-203, https://doi.org/10.1016/ 0004-3702(81)90024-2.

Hwang, Y., A. J. Clark, V. Lakshmanan, and S. E. Koch, 2015: Improved nowcasts by blending extrapolation and model forecasts. Wea. Forecasting, 30, 1201-1217, https://doi.org/ 10.1175/WAF-D-15-0057.1.

Junyent, F., V. Chandrasekar, D. McLaughlin, E. Insanic, and N. Bharadwaj, 2010: The CASA Integrated Project 1 networked radar system. J. Atmos. Oceanic Technol., 27, 61-78, https://doi.org/10.1175/2009JTECHA1296.1.

Kessler, E., 1995: On the continuity and distribution of water substance in atmospheric circulations. Atmos. Res., 38, 109-145, https://doi.org/10.1016/0169-8095(94)00090-Z.

Meinhardt-Llopis, E., J. Sánchez Prez, and D. Kondermann, 2013: Horn-Schunck optical flow with a multi-scale strategy. Image Process. On Line, 3, 151-172, https://doi.org/10.5201/ ipol.2013.20.

Nelder, J. A., and R. Mead, 1965: A simplex method for function minimization. Comput. J., 7, 308-313, https://doi.org/10.1093/ comjnl/7.4.308.

Rabier, F., H. Jrvinen, E. Klinker, J.-F. Mahfouf, and A. Simmons, 2000: The ECMWF operational implementation of four-dimensional variational assimilation. I: Experimental results with simplified physics. Quart. J. Roy. Meteor. Soc., 126, 1143-1170, https://doi.org/10.1002/ qj. 49712656415 .

Rawlins, F., S. P. Ballard, K. J. Bovis, A. M. Clayton, D. Li, G. W. Inverarity, A. C. Lorenc, and T. J. Payne, 2007: The Met Office global four-dimensional variational data assimilation scheme. Quart. J. Roy. Meteor. Soc., 133, 347-362, https://doi.org/ 10.1002/qj.32.

Rinehart, R. E., and E. T. Garvey, 1978: Three-dimensional storm motion detection by conventional weather radar. Nature, 273, 287-289, https://doi.org/10.1038/273287a0.

Roebber, P., 2009: Visualizing multiple measures of forecast quality. Wea. Forecasting, 24, 601-608, https://doi.org/10.1175/ 2008WAF2222159.1.

Rotunno, R., J. B. Klemp, and M. L. Weisman, 1988: A theory for strong, long-lived squall lines. J. Atmos. Sci., 45, 463-485, https://doi.org/10.1175/1520-0469(1988)045<0463: ATFSLL $>2.0 . \mathrm{CO} ; 2$.

Ruzanski, E., and V. Chandrasekar, 2012: Nowcasting rainfall fields derived from specific differential phase. J. Appl. Meteor. Climatol., 51, 1950-1959, https://doi.org/10.1175/ JAMC-D-11-081.1.

Seed, A. W., C. E. Pierce, and K. Norman, 2013: Formulation and evaluation of a scale decomposition-based stochastic precipitation nowcast scheme. Water Resour. Res., 49, 6624-6641, https://doi.org/10.1002/wrcr.20536.

Stam, J., 1999: Stable fluids. SIGGRAPH '99: Proceedings of the 26th Annual Conference on Computer Graphics and Interactive Techniques, ACM Press/Addison-Wesley Publishing Co., 121-128, https://doi.org/10.1145/311535.311548. 
Sun, J., and N. A. Crook, 1997: Dynamical and microphysical retrieval from Doppler radar observations using a cloud model and its adjoint. Part I: Model development and simulated data experiments. J. Atmos. Sci., 54, 1642-1661, https://doi.org/ 10.1175/1520-0469(1997)054<1642:DAMRFD>2.0.CO;2. , and _ 1998: Dynamical and microphysical retrieval from Doppler radar observations using a cloud model and its adjoint. Part II: Retrieval experiments of an observed Florida convective storm. J. Atmos. Sci., 55, 835-852, https://doi.org/ 10.1175/1520-0469(1998)055<0835:DAMRFD>2.0.CO;2.
Tanguay, M., L. Fillion, E. Lapalme, and M. Lajoie, 2012: Fourdimensional variational data assimilation for the Canadian regional deterministic prediction system. Mon. Wea. Rev., 140, 1517-1538, https://doi.org/10.1175/MWR-D-11-00160.1.

Velden, C., and Coauthors, 2005: Recent innovations in deriving tropospheric winds from meteorological satellites. Bull. Amer. Meteor. Soc., 86, 205-223, https://doi.org/10.1175/BAMS-86-2-205.

Wakimoto, R. M., 1982: The life cycle of thunderstorm gust fronts as viewed with Doppler radar and rawinsonde data. Mon. Wea. Rev., 110, 1060-1082, https://doi.org/10.1175/ 1520-0493(1982)110<1060:TLCOTG > 2.0.CO;2. 